\title{
Stable synapsis of homologous DNA molecules mediated by the Escherichia coli RecA protein involves local exchange of DNA strands
}

\author{
Kenji Adzuma \\ Laboratory of Molecular Biology, National Institute of Diabetes, and Digestive, and Kidney Diseases, National Institutes of \\ Health, Bethesda, Maryland 20892 USA
}

Escherichia coli RecA protein promotes stable synapsis between a single-stranded DNA and a homologous duplex DNA, resulting in the formation of a complex of RecA with three DNA strands. To gain insight into the molecular interactions responsible for DNA synapsis, the base-pairing status within the synaptic complex was analyzed by using dimethylsulfate and potassium permanganate as probes. The results indicate that the original base pairs in the parental duplex are disrupted; one strand is displaced and the other strand appears to be involved in Watson-Crick base-pairing with the incoming single-stranded DNA. The state of base-pairing thus resembles that of the end products of strand exchange and not a canonical DNA triple helix involving non-Watson-Crick base-pairing. The results also indicate that this local strand exchange can occur without homology at the ends of the DNA substrates (i.e., when axial rotation of the product heteroduplex with respect to the axis of the parental duplex is obstructed). Taken together, these results suggest that exchange of DNA strands mediated by RecA occur at or before the stage of stable DNA synapsis by a process that does not require DNA rotation.

[Key Words: Homologous recombination; recA protein; DNA synapsis; DNA strand exchange]

Received February 27, 1992; revised version accepted June 22, 1992.

An initial phase of homologous recombination is the exchange of base-pairing partners between two homologous DNAs. The RecA protein of Escherichia coli can promote this DNA strand exchange reaction. An overall scheme for the strand exchange between doublestranded DNA (dsDNA) and single-stranded DNA (ssDNA) mediated by RecA can be depicted as follows (for reviews, see Cox and Lehman 1987; Radding 1988; Kowalczykowski 1991). The initial stage is the polymerization of RecA molecules onto ssDNA in the presence of ATP, resulting in the formation of a helical nucleoprotein filament called the presynaptic complex (Flory et al. 1984; Tsang et al. 1985). The presynaptic complex then captures a naked, homologous dsDNA. Hydrolysis of ATP is not required up to this synapsis stage (Menetski et al. 1990; Rosselli and Stasiak 1990). The final stage is release of the strand exchange products, the displaced ssDNA and a heteroduplex molecule, although the latter product normally stays bound to RecA for a considerable period (Pugh and Cox 1987). One of the roles of ATP hydrolysis may be to facilitate dissociation of RecA from DNA, but without obligatory coupling (Menetski and Kowalczykowski 1985, 1987).

The least understood aspect of the strand exchange reaction is the mechanism by which RecA recognizes homology between two DNAs. This issue is related to another question: At which stage in the reaction does the exchange of DNA strands take place? The simplest model for homology recognition is that it depends entirely on nascent Watson-Crick base-pairing between the ssDNA in the presynaptic complex and the complementary strand in the target dsDNA. This model demands that the original base pairs in the parental dsDNA must be disrupted before the recognition of homology; homology recognition is mechanistically coupled with exchange of DNA strands. Alternatively, the presynaptic complex might recognize a homology without disruption of the original base pairs. One may envision some kind of triplex DNA in which the ssDNA is bonded to the intact dsDNA by, for instance, non-Watson-Crick base-pairing (Howard-Flanders et al. 1984); exchange of DNA strands occurs after homology recognition. In favor of this model, Hsieh et al. (1990) and Rao et al. (1991) reported that RecA can mediate formation of a proteinfree, stable DNA structure whose properties were proposed to be consistent with the stable triplex DNA with additional base-pairings.

Although the exact nature of this interesting DNA structure is yet to be determined, it has become an accepted view that all three strands are held together stably 
within a RecA filament before it releases the strand exchange products. Evidence for this DNA-RecA complex involving three DNA strands, or synaptic complex, has been provided by electron microscopic observation of DNA-RecA complexes at early stages of the strand exchange reaction (Register et al. 1987; Stasiak and Egelman 1987). Recent studies indicating that all three strands can be cross-linked to each other at a presumptive synapsis stage (Bortner and Griffith 1990; Umlauf et al. 1990) also strongly support this view. The synaptic complex appears to accumulate in reactions promoted by the nonhydrolyzable ATP analog, ATPyS /Honigberg et al. 1985). Because the synaptic complex made in the presence of ATP $\gamma$ S yields normal strand exchange products when RecA is removed, the resident DNA structure was proposed to be essentially the end product of strand exchange (Menetski et al. 1990; Rosselli and Stasiak 1990). Alternatively, removal of RecA might have caused a structural transition of some unusual DNA structure into the normal strand exchange products.

To understand the molecular mechanism of DNA synapsis and homology recognition, it is important to analyze directly the base-pairing status within the synaptic complex. Are the original base pairs preserved in the complex or disrupted? To address this issue, the state of base-pairing within the synaptic complex, which was made in the presence of ATP $\gamma S$, was probed by examining the reactivities of the three DNA strands to dimethylsulfate (DMS) and potassium permanganate $\left(\mathrm{KMnO}_{4}\right)$. The results indicate that the base-pairing status within the synaptic complex is essentially the end product of strand exchange.

\section{Results}

For chemical probing analyses of the synaptic complex to be informative, two other issues must also be addressed. First, DNA strands in RecA filaments are known to be conformationally distinct from naked DNAs. It is therefore important to examine, as the reference states for the patterns obtained for the synaptic complex, how simple ssDNA-RecA and dsDNA-RecA complexes react with DMS and $\mathrm{KMnO}_{4}$. Second, one must establish a method to select the synaptic complex out of a reaction mixture that contains various DNARecA complexes and free DNAs.

How do ssDNA-RecA and dsDNA-RecA complexes react with $\mathrm{DMS}$ and $\mathrm{KMnO}_{4}$ ?

DMS primarily methylates $\mathrm{N}-7$ of guanine (G), a position that is protected from the methylation in at least some of the canonical DNA triple helices (Hanvey et al. 1990; Collier et al. 1991) and was also proposed to be involved in a putative RecA-mediated triple helix /Hsieh et al. 1990). DMS also methylates, with lower efficiencies, $\mathrm{N}-3$ of adenine (A) and N-3 of cytosine (C). In the latter case, $\mathrm{C}$ can become accessible to DMS only when it is not base-paired, because the N-3 of C is involved in Wat-
son-Crick base-pairing. $\mathrm{KMnO}_{4}$ oxidizes the C-5/C-6 double bond of thymine (T) (Iida and Hayatsu 1971). Because this oxidation occurs by attack perpendicular to the pyrimidine ring, base-stacking provides protection and normal dsDNA is practically insensitive to $\mathrm{KMnO}_{4}$. When stacking interactions are disrupted, as in the melting of dsDNA, the susceptibility to $\mathrm{KMnO}_{4}$ increases greatly (Hayatsu and Ukita 1967).

How are these features realized in the DNA within simple ssDNA-RecA or dsDNA-RecA complexes? The DNA strands in these complexes adopt a fairly rigid conformation (Takahashi et al. 1989), which is underwound and extended, with a reduced twist $(-20$ bp or bases per helical turn) and with an axial base separation $\sim 1.5$-fold larger than that of normal B-DNA (for review, see Stasiak and Egelman 1988). Previous studies with DMS (Leahy and Radding 1986; DiCapua and Müller 1987) have shown that N-7 of G is susceptible to DMS attack in both ssDNA-RecA and dsDNA-RecA complexes. DiCapua and Müller (1987) also showed that N-3 of C is accessible to DMS in ssDNA-RecA complexes but not in dsDNA-RecA complexes, suggesting that the duplex within a dsDNA-RecA complex retains its original basepairing. These features were confirmed under the conditions used in this study (data not shown).

To test the $\mathrm{KMnO}_{4}$ reactivities, ssDNA-RecA complexes were formed by incubating an end-labeled 40-mer ssDNA $\left(\mathrm{T}_{40}\right.$ or $\mathrm{B}_{40}$; Fig. $\left.1 \mathrm{~A}\right)$ with RecA and ATP $\gamma \mathrm{S}$. The results (Fig. $2 \mathrm{~A}$ ) indicated that reactivities of $\mathrm{T}$ residues in the ssDNA increase with binding to $\operatorname{RecA}$ (the patterns are summarized schematically in Fig. 9A, below). Thus, DNA bases in the ssDNA-RecA complexes appear to be more unstacked than those in naked ssDNAs, probably because of an increase in axial base separation.

Because dsDNA-RecA complexes exhibit an axial polarity (Egelman and Stasiak 1986), incubation of normal dsDNA with RecA would result in a mixture of two complexes with opposite polarities. To make dsDNARecA complex with a single polarity, a partial duplex DNA with a 30-bp-long ssDNA tail attached to one of its $5^{\prime}$ ends was made by annealing a 70 -mer with a 40 -mer ssDNA (duplex 1 or 2 ; Fig. 1B). The presence of a $5^{\prime}$ ssDNA tail in otherwise dsDNA facilitates polymerization of RecA molecules onto the contiguous duplex /Cassuto and Howard-Flanders 1986; Shaner and Radding 1987). In the following text, the strands with a tail are called loading strands, and their complements are called nonloading strands.

$\mathrm{KMnO}_{4}$ reactivities of two strands in each dsDNARecA complex are shown in Figure 2B. In the loading strands (see panels labeled loading), without RecA, only the ssDNA tail (indicated by a bracket) showed a significant sensitivity to $\mathrm{KMnO}_{4}$, whereas the duplex region was virtually insensitive as expected (lanes 1,2 ). In the presence of RecA and ATP $\gamma$ S, however, some T residues in the duplex region also became susceptible to $\mathrm{KMnO}_{4}$ (lanes 3,4 ), whereas, somewhat surprisingly, other $\mathrm{T}$ residues in this region remained completely resistant. The sensitive $T$ residues (solid arrowheads) correspond to all of the $5^{\prime}-\mathrm{TC}-3^{\prime}$ sequences in the duplex region, suggest- 

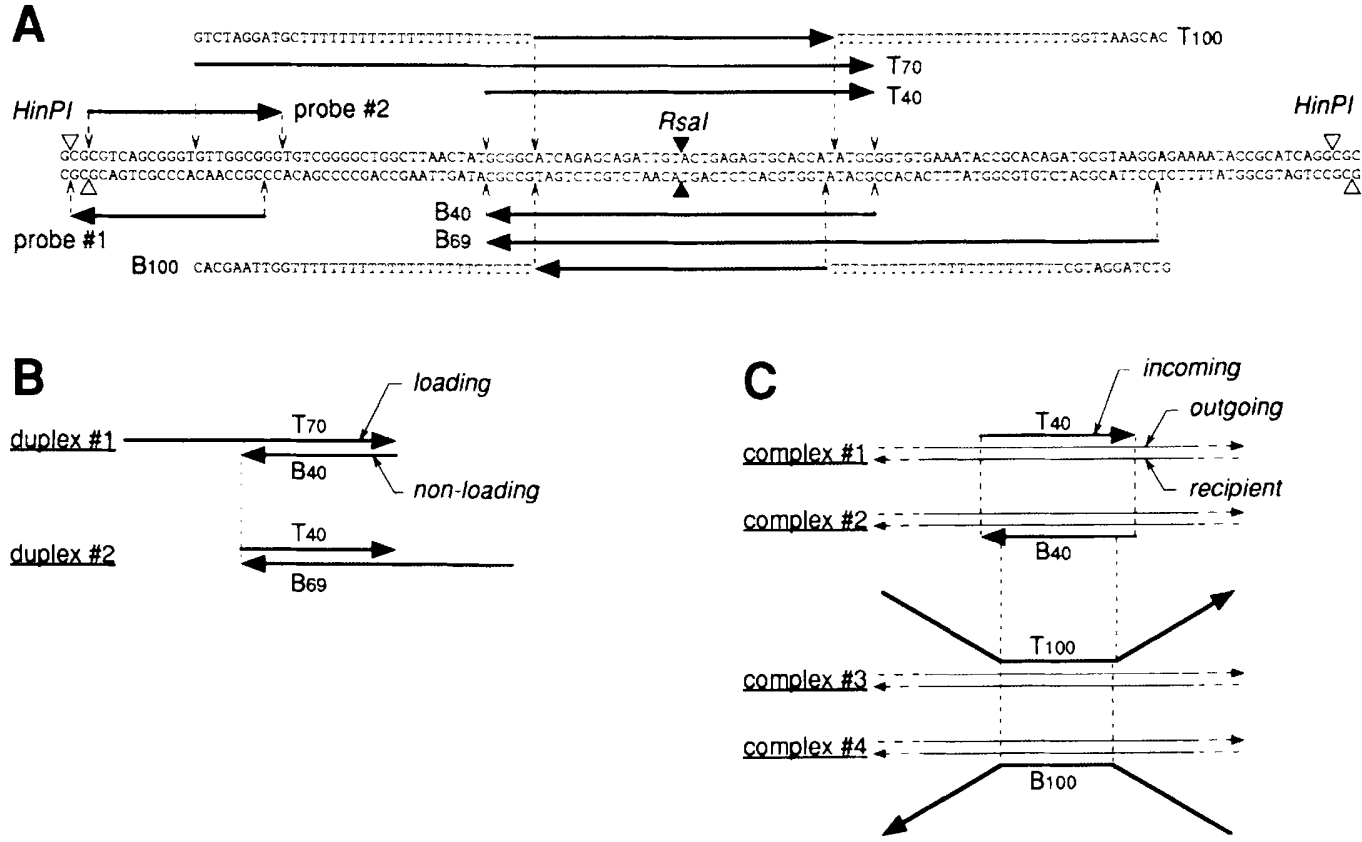

Figure 1. DNA substrates. (A) ssDNAs used in chemical probing analyses. All of the ssDNAs are homologous to a 130-bp HinPI fragment of pUC18 (at position 106-236) that contains one of its Rsal sites. Locations and orientations of the ssDNAs are shown by solid lines with arrowheads indicating their $5^{\prime} \rightarrow 3^{\prime}$ polarities. Except for the hybridization probes $(1$ and 2$)$, the ssDNAs are labeled either $\mathrm{T}$ (identical in sequence to the top strand of the HinPI fragment) or B (bottom strand), with subscripts indicating their lengths. In $T_{100}$ or $B_{100}$, its 30 -base homology is sandwiched by two nonhomologous sequences ( 35 bases in length) as indicated. (B) Duplex DNA substrates for dsDNA-RecA complexes. (Loading strands) Strands with 5 'ssDNA tails; (nonloading strands) their complements. $(C)$ Substrate combinations for synaptic complexes. Thin lines indicate the target duplexes; thick lines indicate the incoming ssDNA strands. (Outgoing strands) Strands identical in sequence to incoming strands; (recipient strands) strands complementary to incoming strands.

ing a particularly weak stacking between these two bases in this orientation (for sequence, see Fig. 1A; neither $T$ of CT nor T of TT was sensitive). The reactivities of nonloading strands (Fig. 2B, panels labeled nonloading) were related to, but at the same time distinct from, those of loading strands; they were related in that the reactivity at each $\mathrm{T}$ was position dependent, showing a preference for $\mathrm{T}$ of $\mathrm{TC}$, but were distinctive in that this variability was much less in the nonloading strands /see also Fig. 9B, below for the schematic diagrams). In brief, the duplex DNA in dsDNA-RecA complexes is generally more reactive to $\mathrm{KMnO}_{4}$ than naked dsDNA, although the base pairs in dsDNA-RecA complexes are probably not disrupted (DiCapua and Müller 1987). The possible conformational asymmetry between two strands in dsDNARecA complexes was also noted in studies with nucleases as probes (Chow et al. 1988; Lindsley and Cox 1989).

\section{Formation and characterization of the synaptic complex}

In this study the synaptic complexes were made by incubating pUC18 dsDNA /linearized by the restriction enzyme ScaI unless otherwise noted) and short ssDNA in the presence of RecA and ATP $\gamma$ S. The homology 130 or $40 \mathrm{bp}$ ) was restricted to the middle of the linear dsDNA, a region that includes one of the RsaI restriction sites of
pUC18. In the presence of optimal concentrations of RecA (see below), only this particular RsaI site becomes protected from RsaI digestion. As described later, chemical reactivity patterns of the synaptic complexes were obtained from the DNA that survived the RsaI digestion. The experiments described below show that the restriction enzyme-resistant entity requires a homologous ssDNA for its formation, contains the ssDNA and RecA, and can be chased into the normal strand exchange product, thus satisfying the criteria for the synaptic complex.

Figure 3A (lanes 2-6) shows that the protection of particular RsaI sites is dependent on homologous ssDNA. The RsaI-resistant entity, once formed, appears to be very stable; although the $R s a I$ added was enough to completely cleave naked pUC18 DNA within 3 min and retained activity even after a l-hr incubation /data not shown), a longer digestion with $R s a$ I had almost no effect on the band intensity of the specific partial digest, B + C (Fig. 3A, cf. lane 7 with lane 4). The RsaI-resistant entity, however, rapidly became sensitive to $R s a I$ upon adding an excess of challenging, nonhomologous ssDNA $(\phi \mathrm{X}$ viral DNA) (Fig. 3A, cf. lane 10 with lane 4), probably owing to rapid transfer of RecA molecules from the complex to the $\phi \mathrm{X}$ ssDNA. This result indirectly ensures that the RsaI-resistant entity was a DNA-RecA complex and not a protein-free DNA structure that was somehow resistant to RsaI. 

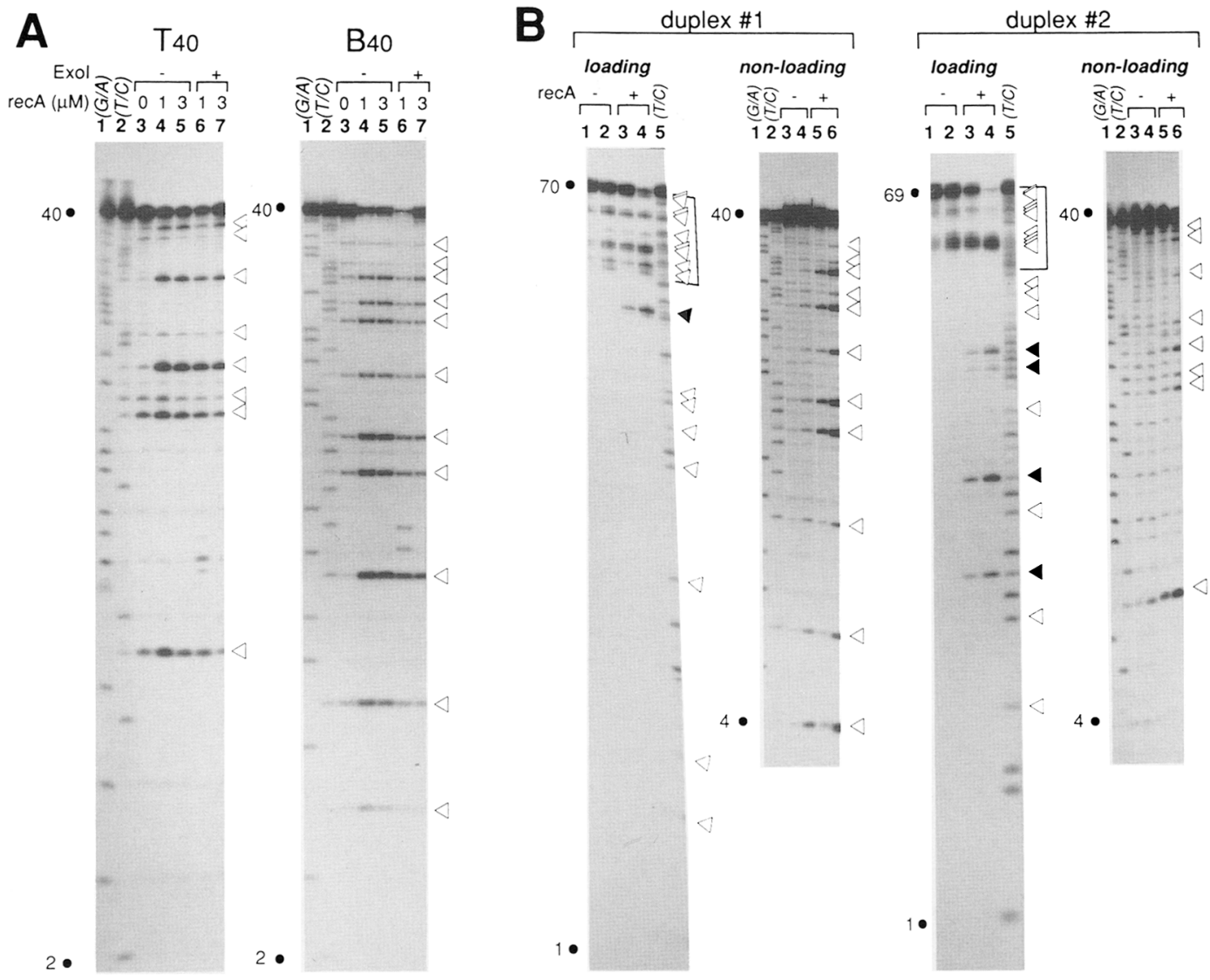

Figure 2. $\mathrm{KMnO}_{4}$ reactivities of ssDNA-RecA and dsDNA-RecA complexes. $(A)$ ssDNA-RecA complexes. DNA substrate, either $\mathrm{T}_{40}$ or $\mathrm{B}_{40}\left(0.74 \mu \mathrm{M}\right.$, in nucleotide); $\mathrm{KMnO}_{4}$ concentration, $0.6 \mathrm{~mm}$. (Arrowheads) Positions of $\mathrm{T}$. (Lanes 1,2$) \mathrm{Maxam}-\mathrm{Gilbert} \mathrm{G}+\mathrm{A}$ and $T+C$ ladders; (lanes $3-5$ ) reactivities at 0,1 , and $3 \mu \mathrm{M}$ of RecA; (lanes 6,7 ) same as lanes 4,5 , except that the samples were incubated with Exo I before chemical modification. $(B)$ dsDNA-RecA complexes. The duplex DNA substrates (duplexes 1 and $2, \sim 2.2 \mu \mathrm{M}$ in nucleotide) are indicated (top), each of which contains two panels corresponding to the loading strand (labeled at the 3 ' end) and nonloading strand (labeled at the $5^{\prime}$ end). (Arrowheads) Positions of $T$ (sensitive $T$ residues in the duplex region of loading strands are marked by solid arrowheads); (brackets) ssDNA tail regions. Panels of loading strands: (Lanes 1,2 and 3,4) Without and with $1.8 \mu \mathrm{M}$ RecA, respectively; (lane 5) T + C ladder. $\mathrm{KMnO}_{4}$ concentration: (Lanes 1,3) $0.4 \mathrm{~mm}$; (lanes 2,4) 1.2 mM. Panels of nonloading strands: (Lanes 1,2) G + A and T + C ladders; (lanes 3,4 and 5,6), without and with $1.8 \mu \mathrm{M} \mathrm{RecA}$, respectively. $\mathrm{KMnO}_{4}$ concentration: (lanes 3,5) $1.2 \mathrm{~mm}_{i}$ (lanes 4,6) $4.0 \mathrm{~mm}$. Minor bands at positions other than $\mathrm{T}$ in the nonloading strands were the result of nonspecific cleavage by piperidine; for some unknown reason, these synthesized oligonucleotides were susceptible to random piperidine cleavage even without base modification.

The rapid transfer of RecA was also observed at a lower concentration of RecA (Fig. 3B, lanes 1-6), whereas at the higher concentration (lanes 7-12) roughly one-third of the homology-dependent, RsaI-resistant complex remained resistant to the RsaI, even in the presence of the challenging ssDNA (cf. lanes 9 and 10 with lane 8). Use of five times more of the $\phi \mathrm{X}$ ssDNA did not affect the results (data not shown). Thus, it is possible that, depending on the RecA concentration/and/or stoichiometry), two kinds of RsaI-resistant complexes might exist with respect to the insensitivity to challenging ssDNAs. Note that band intensity of the full-length linear $(A+B+C)$, which presumably represents a patch of RecA molecules bound nonspecifically and/or those owing to contiguous polymerization from the synaptic complex, was also insensitive to the ssDNA challenge (Fig. 3B, lanes 9,10$)$. This observation suggests that RecA mole- cules involved in the challenging ssDNA-sensitive complex, hereafter referred to as synaptic complex, may be in a state different from those involved in simple dsDNARecA complexes.

In chemical probing analyses of the synaptic complex, the RecA concentration was optimized so that (1) the band intensity of the corresponding RsaI partial digest $(B+C)$ was maximum, (2) those of full-length linear $(\mathrm{A}+\mathrm{B}+\mathrm{C})$ and the other partial digest $(\mathrm{A}+\mathrm{C})$ were negligible, and (3) most of the complex was sensitive to the ssDNA challenge.

The synaptic complex is an intermediate of strand exchange

The synaptic complex can produce a D-loop when RecA is removed, which satisfies a criteria as an intermediate 
A

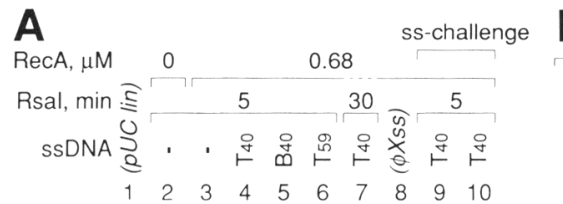

$\mathbf{B}_{\text {RecA, } 0.36 \mu \mathrm{M}}$
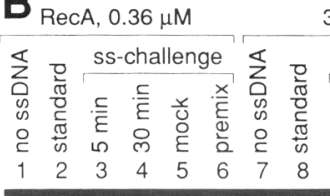

$3.2 \mu \mathrm{M}$

ss-challenge
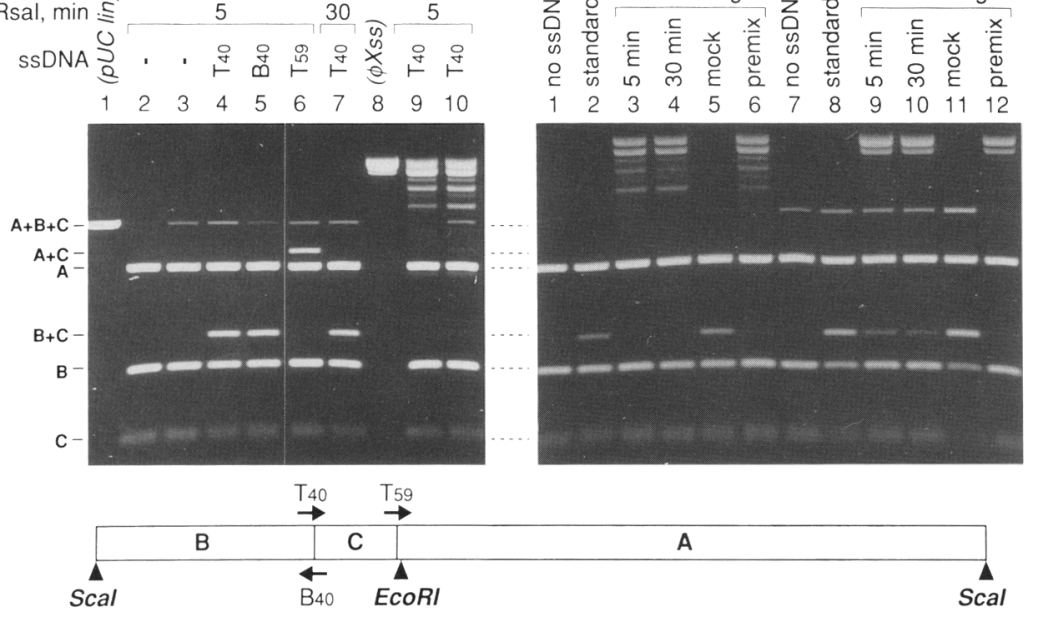

Figure 3. Characterization of synaptic complex. (A) RsaI digestion assay of the synaptic complex. Linear pUC1 8 dsDNA (50 $\mu \mathrm{M}$ in nucleotide) was incubated with various short ssDNA $10.74-1.1$ $\mu \mathrm{M}$, depending on the length), with the molecular ratio of $1: 2$, in the presence of $\operatorname{RecA}(0.68 \mu \mathrm{M})$ and ATP $y S$ for $1 \mathrm{hr}$. The restriction enzyme RsaI was then added to the mixture, and the incubation was continued for another $5 \mathrm{~min}$, followed by analysis of the digestion patterns by agarose gel electrophoresis. (Lanes 1-7) Normal RsaI digestion assays. (Lane 1) Linear pUC18 used as the target duplex; (lane 2) reaction without RecA and short ssDNA; (lane 3) without ssDNA but with RecA; (lanes 4-6) complete reactions with various ssDNA as indicated (see the RsaI restriction map shown at bottom); (lane 7) same as lane 4 except that RsaI digestion was carried out for 30 min. (Lanes $8-10$ ) ssDNA challenge assays. (Lane 10) After the complex formation was completed by a 1 -hr incubation, $\phi \mathrm{X}$ ssDNA was added to

this mixture and the incubation was continued for another $5 \mathrm{~min}$, followed by RsaI digestion; (lane 8) $\phi \mathrm{X}$ viral DNA used as the challenging ssDNA; (lane 9) premix control in which the $\phi X$ ssDNA was added before addition of RecA to start synaptic complex formation. $(B)$ Further ssDNA challenge assays at two different concentrations of RecA: $0.36 \mu \mathrm{M}$ (lanes 1-7) and 3.2 $\mu \mathrm{M}$ (lanes 7-12). Also shown is a normal assay without (lanes 1,7) or with (lanes 2,8 ) short ssDNA substrate $T_{40}$. After the synaptic complex was formed $\phi X$ ssDNA was introduced to this mixture and the incubation was continued for another 5 min (lanes 3,9) or 30 min (lanes 4,10) followed by RsaI digestion; (lanes 5,11) mock treatment in which TE buffer was added instead of $\phi \mathrm{X}$ ssDNA and incubated for another $30 \mathrm{~min}$, followed by Rsal digestion; (lanes 6,12 ) premix control in which $\phi \mathrm{X}$ ssDNA was added before starting the synaptic complex formation.

of strand exchange. For these experiments, the complex was made by use of the ${ }^{32} \mathrm{P}$-labeled short ssDNA $\left(\mathrm{T}_{59}\right)$, which covers the single EcoRI site of pUCl8, and a supercoiled pUC18 DNA (see drawing at the bottom of Fig. 3). After the complex formation, the reaction mixtures were subjected to $E c o$ RI digestion and deproteinized, and the samples were run on an agarose gel. The autoradiogram revealed a band that comigrated with the supercoiled pUC18 (Fig. 4A, lane 4). Approximately half of the EcoRI-resistant complex was calculated to be converted into this species, whereas the rest presumably fell apart. The stable protein-free joint molecule was not obtained in reactions with linear pUC18 [Scal digested; Fig. 4A, lane 8), although the linear form is a competent target in the formation of the synaptic complex. Nor was the joint molecule detected when the supercoiled pUC18 was linearized (by ScaI) after formation of the complex (Fig. 4A, lane 6). These results indicate that the joint molecule requires supercoiling for its stability, which is consistent with the known properties of $D$ loops.

To confirm that these protein-free joint molecules were $\mathrm{D}$ loops, the joint molecule was purified away from free ssDNA substrate and resubjected to $E c o$ RI digestion. Although EcoRI did not digest the free ssDNA (Fig. 4B, solid squares), the ssDNA within the joint molecule (open triangles) was cleaved by EcoRI almost as efficiently as was normal duplex DNA (solid circles). This joint molecule is thus probably not a stable triple helix in which the third strand is hydrogen-bonded to the major groove of intact dsDNA, because this type of structure, as has been the case for canonical DNA triple he- lices (Maher et al. 1989; Hanvey et al. 1990), should resist the digestion by EcoRI that primarily binds to the major groove of its recognition site. Taken together with the supercoiling dependence described above, these results indicate that the majority of joint molecules are simple D loops, the normal product of strand exchange.

\section{The original base pairs are disrupted in the synaptic complex}

To examine the DMS reactivities of the target duplex within the synaptic complex, the complex was formed between linear pUC18 DNA and a short ssDNA that includes one of the RsaI sites in pUC18. Two complementary ssDNAs, $\mathrm{T}_{40}$ and $\mathrm{B}_{40}$, were used in the experiments (these strands are hereafter called incoming strands; see Fig. IC and the legend). The pattern of the synaptic complex was selected by virtue of its $R s a$ I resistance as follows. The reaction mixture containing the complex was digested with $R s a \mathrm{I}$ and then modified with DMS. After quenching and deproteinization, the samples were digested further with the restriction enzyme HinPI. The 130-bp HinPI fragment containing the protected RsaI site (see Fig. 1A) was isolated and chemically cleaved by piperidine at the positions of the methylated bases. The samples were run on a sequencing gel, which was subsequently transferred to a membrane filter. The cleavage pattern was visualized by an indirect end-labeling method: hybridization with either of two ${ }^{32} \mathrm{P}$-labeled probes that hybridize to the end of each strand of the HinPI fragment (probe 1 or 2 ; see Fig. IA). In this way 
A

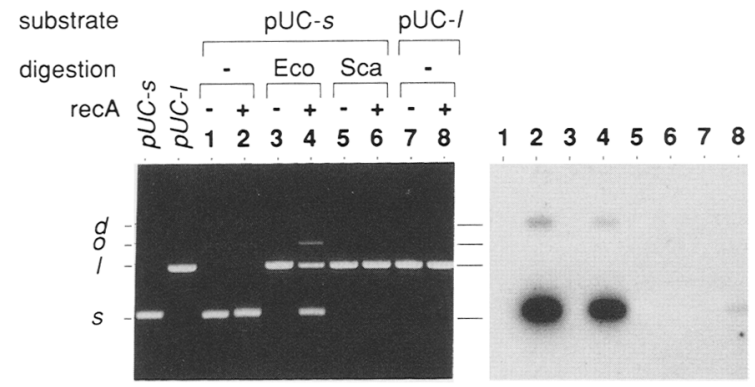

B

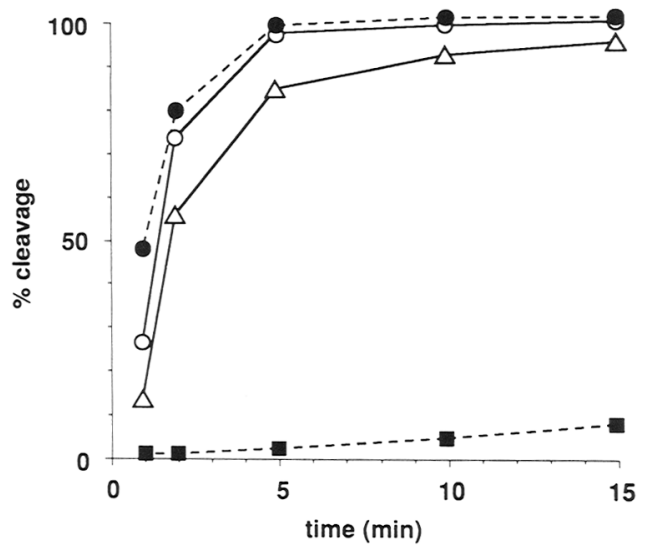

Figure 4. Characterization of D loop. $(A \mid$ Dependence on supercoiling. The synaptic complex was made between the ${ }^{32} \mathrm{p}$. labeled $\mathrm{T}_{59}$, which is homologous to a region containing the single EcoRI site of pUCl 8 (see drawing at the bottom of Fig. 3), and a pUC18 DNA, which was either in a supercoiled form (lanes 1-6) or in a Scal-linearized form (lanes 7,8). Lanes of odd numbers are no RecA controls. After the complex formation, some samples were subjected to digestion with EcoRI (lanes 3,4) or ScaI (lanes 5,6). The samples were analyzed by agarose gel electrophoresis. A picture of the ethidium-stained gel and its autoradiograph are shown at left and right, respectively. Positions of various forms of pUC18 in the gel are indicated by symbols: (s) Supercoiled; (1) linear; (o) open circle; (d) dimer supercoiled. $(B)$ EcoRI digestion kinetics of stable joint molecules. The joint molecule made under the condition of lane 2 in $A$ was purified away from free ssDNA, and the digestion kinetics with EcoRI was analyzed as described in Materials and methods. (O) Cleavage of the radioactive species comigrating with supercoiled pUC18 on agarose gel electrophoresis; $(\triangle)$ Cleavage of labeled $\mathrm{T}_{59}$ within joint molecules as assayed on sequencing gel electrophoresis; $(\mathbf{0}, \mathbf{\square}$, and broken lines) cleavage of control dsDNA (pBR322 supercoiled) and free $\mathrm{T}_{59}$, respectively.

one can compare the pattern between two strands of the target duplex, using exactly the same sample. Also note that the experiments employed the two synaptic complexes that are identical in the region of homology but differ in polarities of the incoming strands /complexes 1 and 2 ; see Fig. 1C). This allows one to compare the pattern between a strand in the target duplex that is identical in sequence to the incoming strand (hereafter called outgoing strands; see legend to Fig. 1) and a strand that is complementary (recipient strand), with exactly the same sequence.

The resulting autoradiogram revealed that certain $C$ residues in the outgoing strands (labeled out in Fig. 5), but not those in the recipient strands (rec), were susceptible to DMS. Almost all sensitive $C$ residues were located in the region homologous to the incoming ssDNAs (indicated by a bracket). These "melted" C residues (solid arrowheads) do not seem to represent only a minor fraction of the complex; the band intensities at these $\mathrm{C}$ residues were $-5-20 \%$ of those at the $G$ residues, depending on the locations, which was similar to the ratio observed in DMS methylation of ssDNAs with or without RecA (an example of the naked ssDNA pattern can be seen below in Fig. 7A, lane 3; see also DiCapua and Müller 1987). Therefore, the original base pairs are mostly disrupted in the synaptic complex, in which the recipient strand appears to be involved in nascent basepairing.

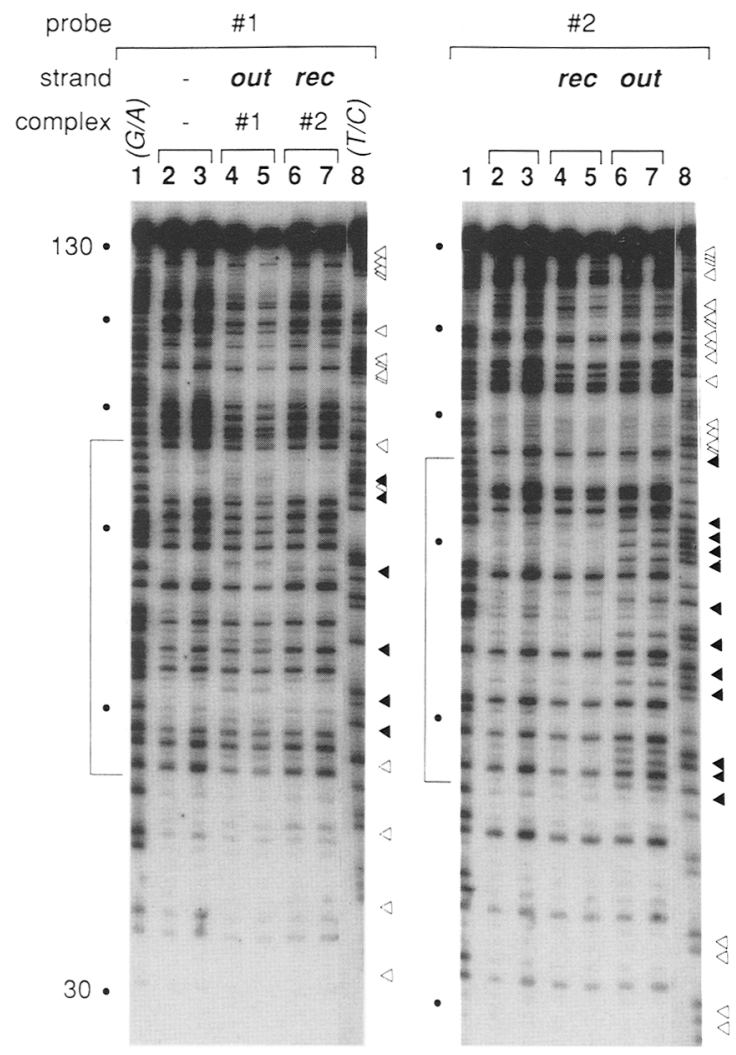

Figure 5. DMS reactivities of outgoing and recipient strands in the synaptic complex. In addition to no RecA, no ssDNA control (lanes 2,3), a set of experiments included synaptic complexes 1 (lanes 4,5) and 2 (lanes 6,7) as indicated (see Fig. 1C). (Lanes 1,8$) \mathrm{G}+\mathrm{A}$ and $\mathrm{T}+\mathrm{C}$ ladders. DMS concentrations: $0.16 \%$ (lanes $2,4,6$ ) and $0.31 \%$ (lanes 3,5,7). The same membrane was probed with each of two complementary probes (1 and 2, left and right, respectively; also see Fig. 1). (Arrowheads) Positions of $\mathrm{C}$ /sensitive $\mathrm{C}$ residues found in the outgoing strands are marked by solid arrowheads); (brackets) regions homologous to the incoming ssDNAs. The major bands correspond to N-7 of G, whereas the minor bands, other than $\mathrm{C}$, correspond to N-3 of A. To aid comparison, each DNA strand is labeled with its functional name, outgoing (out) or recipient (rec) strand, as defined in the legends to Fig. 1. 
There were no obvious differences in DMS susceptibilities at N-7 of G among outgoing strands, recipient strands, and naked dsDNA controls (see the major bands in Fig. 5), suggesting that the N-7 of G of the target duplex is not involved in stable hydrogen bonding within the synaptic complex. This result could be misleading if the majority of $\mathrm{G}$ methylation occurred after destruction of the complex. The following observations /data not shown), however, argue against this possibility. First, as DMS is notoriously difficult to quench thoroughly, great care was taken to process the samples as quickly as possible. Under the conditions used, when DMS was added after addition of DMS stop solution, the degree of G methylation was only $10-20 \%$ of that found with the normal order of addition, indicating that the majority of the methylation occurred before the addition of DMS stop solution. Second, DMS itself did not induce a significant destruction of the complex, as assayed by RsaI challenge. It should also be recalled here that methylation at $\mathrm{C}$ differed between outgoing and recipient strands in exactly the same samples. Because the target dsDNA was linear, if the complex had been completely destroyed during methylation, the target duplex should have restored the original duplex state; in this case, $C$ in both strands should have become equally resistant to DMS.

\section{$\mathrm{KMnO}_{4}$ reactivities of the outgoing and recipient strands are distinctive}

Similar experiments were carried out with $\mathrm{KMnO}_{4}$ as a probe. $\mathrm{KMnO}_{4}$ did not oxidize $\mathrm{T}$ in the naked dsDNA control under these conditions (Fig. 6, lanes 3,4), whereas in the synaptic complex, both outgoing (labeled out) and recipient (rec) strands were sensitive to $\mathrm{KMnO}_{4}$. Most of the sensitive $\mathrm{T}$ residues were located in the region homologous to the ssDNAs (indicated by a bracket), although the effects extended several bases to either side. There is, however, a significant difference in the pattern between outgoing and recipient strands. Although the $T$ residues in outgoing strands showed fairly smooth and uniform susceptibilities, those in the recipient strands displayed a significant position-dependent variability in their reactivities and were generally less sensitive (see also Fig. 9C, below). These results indicate that a severe conformational distortion of the target duplex takes place within the synaptic complex, presumably the unstacking of bases. The results also indicate that outgoing strands may be conformationally distinct from recipient strands in this complex. Taking into account that N-3 of $\mathrm{C}$ is susceptible to DMS only in outgoing strands, the smooth and uniform reactivities shown by $\mathrm{T}$ in this strand to $\mathrm{KMnO}_{4}$ are most probably attributable to its being single stranded, a characteristic not exhibited by the recipient strands.

The $\mathrm{KMnO}_{4}$ reactivity patterns of outgoing strands may look reminiscent of the patterns exhibited by the ssDNA within simple ssDNA-RecA complexes, rather than those of the duplex DNA strands in dsDNA-RecA

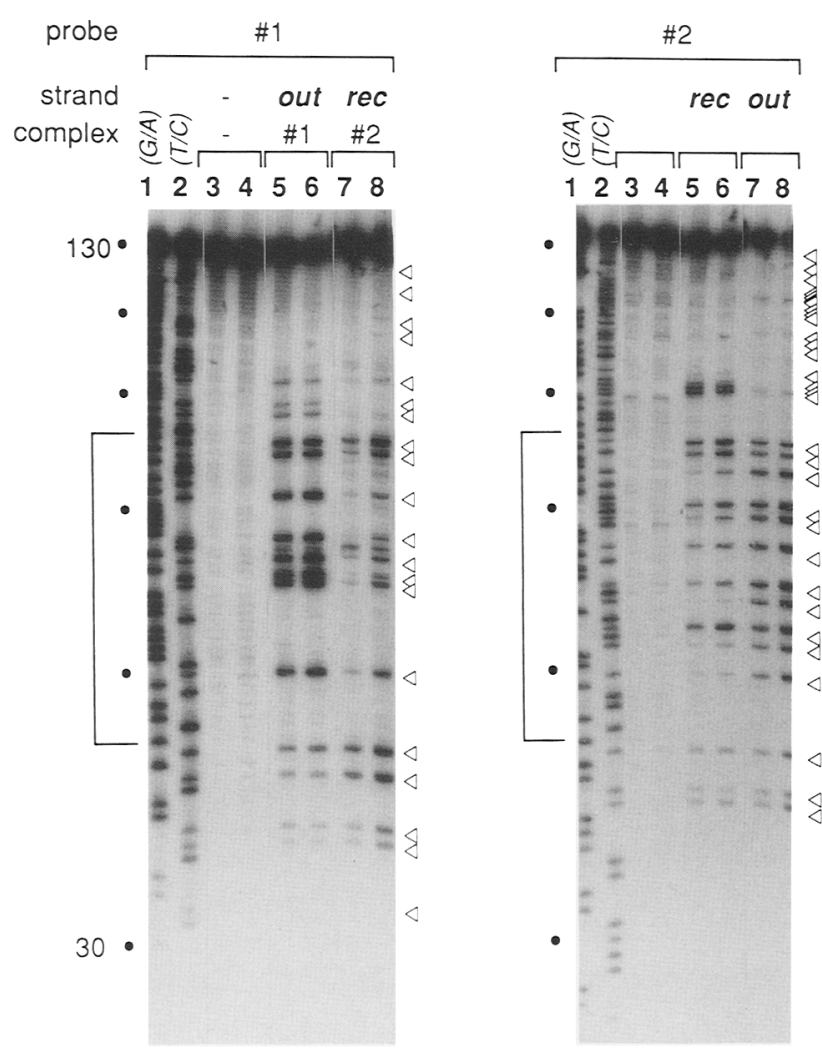

Figure 6. $\mathrm{KMnO}_{4}$ reactivities of outgoing and recipient strands in the synaptic complex. Essentially the same experiments as in Fig. 5 were performed with $\mathrm{KMnO}_{4} . \mathrm{KMnO}_{4}$ concentrations: $0.75 \mathrm{~mm}$ (lanes $3,5,7)$ and $2.5 \mathrm{~mm}$ (lanes $4,6,8$ ). Symbols and labels are the same as in Fig. 5 (except for arrowheads, which indicate positions of $\mathrm{T}$ ).

complexes (see Fig. 9, below); beyond this relative resemblance, however, the difference in $\mathrm{KMnO}_{4}$ reactivity between the outgoing strands and ssDNAs within simple ssDNA-RecA complexes is noticeable. The patterns of recipient strands are qualitatively similar to those of the nonloading strands of dsDNA-RecA complexes; some T residues were more sensitive than others in both strands, and the locations of these sensitive and insensitive $T$ residues agreed with each other reasonably well (cf. stippled bars between Fig. 9B and C).

\section{Chemical reactivities of the incoming and recipient strands are similar}

Chemical probing analyses of incoming strands were carried out with the synaptic complex formed between the ${ }^{32} \mathrm{P}$-labeled ssDNA, $\mathrm{T}_{40}$ or $\mathrm{B}_{40}$ and supercoiled pUC18. The synaptic complex was treated with $\mathrm{KMnO}_{4}$ or DMS and quenched, followed by the removal of RecA. The resulting $\mathrm{D}$ loop was purified away from the free ssDNA, and the sample was then chemically cleaved and analyzed by sequencing gel electrophoresis.

As in the recipient strands, the $\mathrm{N}-3$ of $\mathrm{C}$ in the incom- 
ing strands was not susceptible to DMS methylation (Fig. 7A, lane 5). Under the same condition, $C$ in naked ssDNAs was sensitive to DMS (lane 3), whereas that in the ssDNA within protein-free D loops was not sensitive (lane 4). Reactivity at N-7 of G in the incoming strands was again indistinguishable from that in naked ssDNAs or in the ssDNA within D-loops. These results are consistent with the idea that the incoming and recipient strands are Watson-Crick base paired in the synaptic complex.

The $\mathrm{KMnO}_{4}$ reactivities of the incoming strands (Fig. $7 \mathrm{~B}$, lane 6) also resemble those of recipient strands, in that they both showed a similar position-dependent variability (e.g., c.f. "in" of complex 2 and "rec" of complex 1 in Fig. 9C, below). In contrast, the patterns of the incoming strands were different from those of naked ssDNAs (Fig. 7B, lane 3), the ssDNA within protein-free D loops (lane 4), or the ssDNA within ssDNA-RecA complexes (lane 5). Note that the patterns of incoming strands were obtained by first treating the complexes with a chemical and, after deproteinization, purifying the resulting $\mathrm{D}$ loops; no a priori reason exists to believe that the very positions of modified bases did not influence the efficiency of formation or stability, or both, of the protein-free D-loops. With this caveat in mind, however, the results shown in Figure 7 nonetheless indicate that the chemical reactivities of incoming and recipient strands resemble each other. Also note that both of these patterns are, in turn, similar to those exhibited by the nonloading strands in dsDNA-RecA complexes (see Fig. 9 , below!.

Presence or absence of a homologous end does not affect the base-pairing status within the synaptic complex

Exchange of DNA strands has been viewed sometimes as the unwinding of a parental duplex and the concomitant rewinding of a heteroduplex. According to this view, strand exchange is, in effect, coupled with axial rotation of the heteroduplex with respect to the axis of the parental duplex. This unwinding-rewinding of DNA strands requires a combination of DNA substrates that share a homology at the ends of DNAs. Because formation of the synaptic complex itself does not require the homologous DNA end (DasGupta et al. 1980; Bianchi et al. 1983), it has been speculated that the synaptic complex consisting of the DNA substrates without a homologous end might represent an interesting intermediate before actual exchange of DNA strands. The following experiments examine the structure of the synaptic complex lacking a homologous DNA end, to compare it with the complex carrying homologous ends that has been analyzed so far.

To make the complex lacking a homologous end, a
Figure 7. Chemical reactivities of the incoming strand in the synaptic complex. The synaptic complex was made between supercoiled pUC18 and $5^{\circ}$. labeled $\mathrm{T}_{40}$ or $\mathrm{B}_{40}$. $(A)$ DMS reactivities (concentration, $0.25 \%$ ). (Arrowheads) Positions of C. (Lanes 1,2) $\mathrm{G}+\mathrm{A}$ and $\mathrm{T}+\mathrm{C}$ ladders; (lane 3) naked ssDNA control; (lane 4) ssDNA within the proteinfree $D$ loop that was purified without DMS modification and then treated with DMS; (lane 5) incoming ssDNA within the synaptic complex. $(B) \mathrm{KMnO}_{4}$ reactivities (concentration, $0.75 \mathrm{~mm}$ ). (Arrowheads) Positions of $T$. (Lanes 1,2) $\mathrm{G}+\mathrm{A}$ and $\mathrm{T}+\mathrm{C}$ ladders; (lane 3) naked ssDNA control; (lane 4) ssDNA within the protein-free D loop; (lane 5) ssDNA within the simple ssDNA-RecA complex; (lane 6) incoming ssDNA within the synaptic complex.

40

A
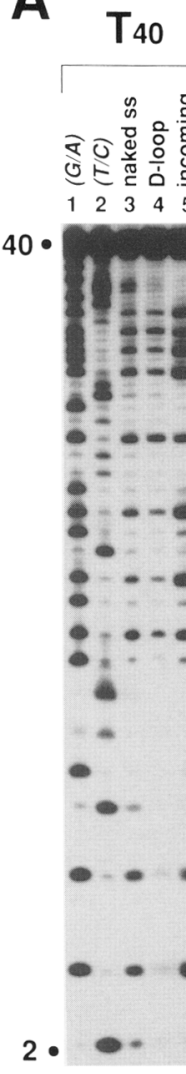$$
\begin{array}{lllll}
2 & 3 & 4 & 5
\end{array}
$$

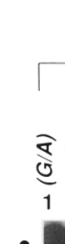

$\mathrm{B}_{40}$

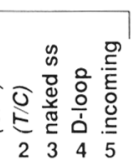

2 至

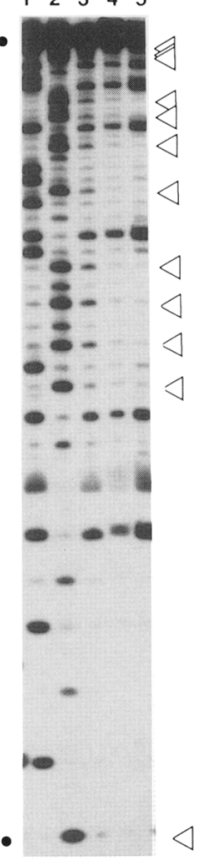

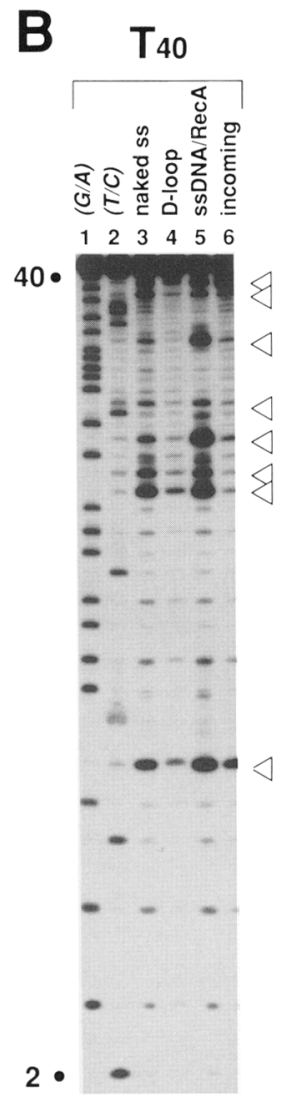

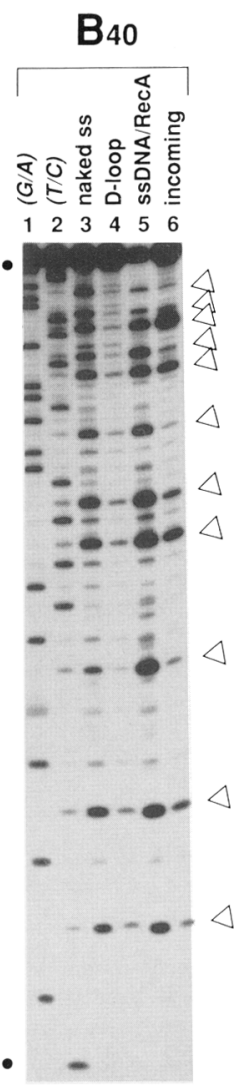


100-mer ssDNA ( $\mathrm{T}_{100}$ or $\mathrm{B}_{100}$; see Fig. $1 \mathrm{~A}$ ), in which only the middle 30 -bp sequence is homologous to the middle of the parental duplex, was used as the incoming strand (complex 3 or 4 , see Fig. 1C). These partially homologous ssDNAs did form the synaptic complexes efficiently, as assayed by RsaI challenge. Nonetheless, the resulting synaptic complexes were unproductive as expected; the complex could not be chased into a stable $D$ loop upon removal of RecA, even if the target dsDNA was supercoiled (data not shown).

Chemical probing of the target duplexes in these complexes was carried out by exactly the same protocol as described for the complexes carrying homologous ends, and the results are shown in Figure 8, A (DMS) and B $\left(\mathrm{KMnO}_{4}\right)$. As with the complex carrying homologous ends, the N-3 of $\mathrm{C}$ in the outgoing strand (labeled out in Fig. $8 \mathrm{~A}$ ), but not in the recipient strand (rec), was susceptible to DMS attack; N-7 of G remained accessible in both strands. Both outgoing and recipient strands became sensitive to $\mathrm{KMnO}_{4}$, with the recipient strand showing much more uneven reactivity than the outgoing strand (Fig. $8 \mathrm{~B}$ ). The $\mathrm{KMnO}_{4}$ pattern of complex 1 (carrying homologous ends; see Fig. 6) and that of complex 3 shown in Figure 8B (lacking a homologous end) are essentially identical (see Fig. 9C). A subtle difference between the two complexes can only be seen outside the homologous regions, where the complex with homologous ends exhibited gradual decrease in sensitivities to $\mathrm{KMnO}_{4}$; the sensitive Ts in the complex lacking a homologous end were confined much more to the homologous regions. In brief, there was no obvious difference in either DMS or $\mathrm{KMnO}_{4}$ reactivity between the two types of synaptic complexes in the region of homologous contacts.

\section{Discussion \\ Stable DNA synapsis is preceded by or occurs simultaneously with exchange of DNA strands}

Chemical probing of base-pairing status within the synaptic complex indicates that the original bair pairs in the target duplex are disrupted. The outgoing strand, which would eventually be displaced, is already single stranded in the complex. First, N-3 of C, a hydrogen-bonding acceptor in Watson-Crick base-pairing, is sensitive to DMS in this strand. Second, $\mathrm{KMnO}_{4}$ reactivities of $\mathrm{T}$ residues in this strand are, as a whole, distinctively smooth and uniform. In contrast, the recipient strand appears to be involved in nascent base-pairing with the incoming strand in the complex. In support of this idea, $\mathrm{C}$ in both the recipient and incoming strands is insensitive to DMS. Also, $\mathrm{T}$ residues in these strands do not show such smooth and uniform sensitivities to $\mathrm{KMnO}_{4}$ as they do in outgoing strands but, instead, exhibit a pattern that is qualitatively similar to that of the nonloading strand in simple dsDNA-RecA complexes. Taking into account that N-7 of $G$ is not stably protected from DMS attack in any of the three strands in the synaptic complex, these results taken together support the view that the base-pairing status in the synaptic complex is essentially that of the end product of strand exchange, displaced ssDNA (outgoing strand) plus heteroduplex (a duplex comprising incoming and recipient strands). From a mechanistic point of view, this means that exchange of DNA strands takes place at or before the stable synapsis stage that the synaptic complexes analyzed here represent.

A caveat inherent in chemical probing analyses of protein-DNA complexes in general must be noted; these
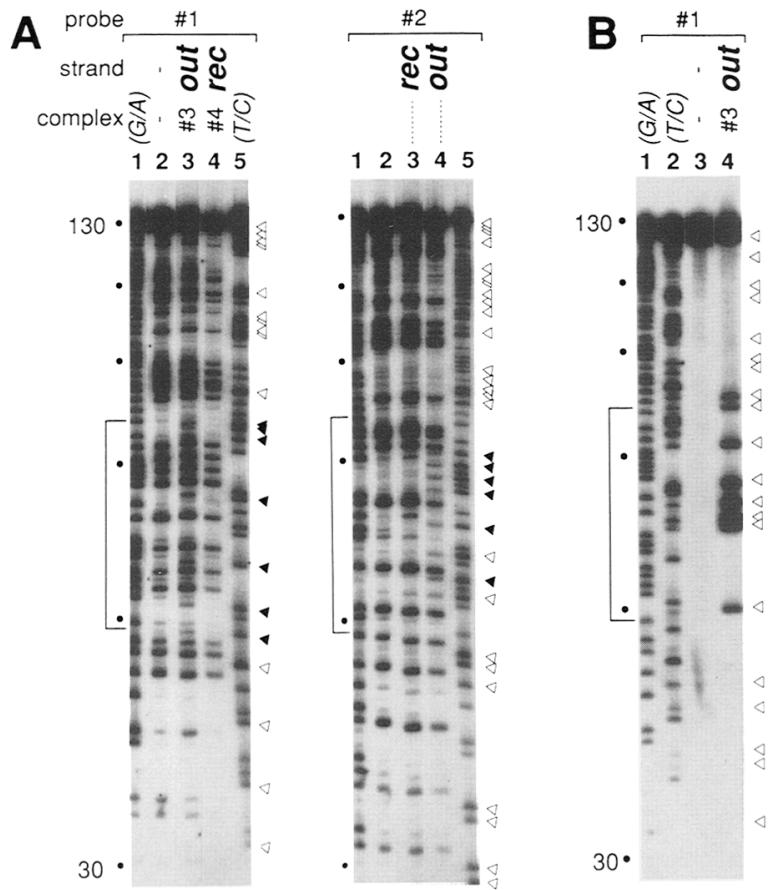

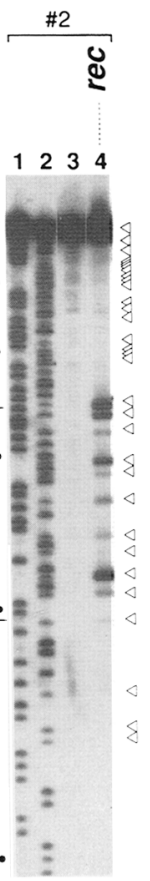

Figure 8. Chemical reactivities of the synaptic complex without a homologous end. The synaptic complexes analyzed here are complexes 3 and 4 (see Fig. 1C). (A) DMS reactivities (concentration, $0.31 \%$ ); $(B)$ $\mathrm{KMnO}_{4}$ reactivities (concentration, $2.5 \mathrm{~mm}$ ). (Arrowheads) Positions of $C$ and $T$ in $A$ and $B$, respectively (solid arrowheads in $A$ indicate the positions of sensitive $C$ residues found in the outgoing strands). 


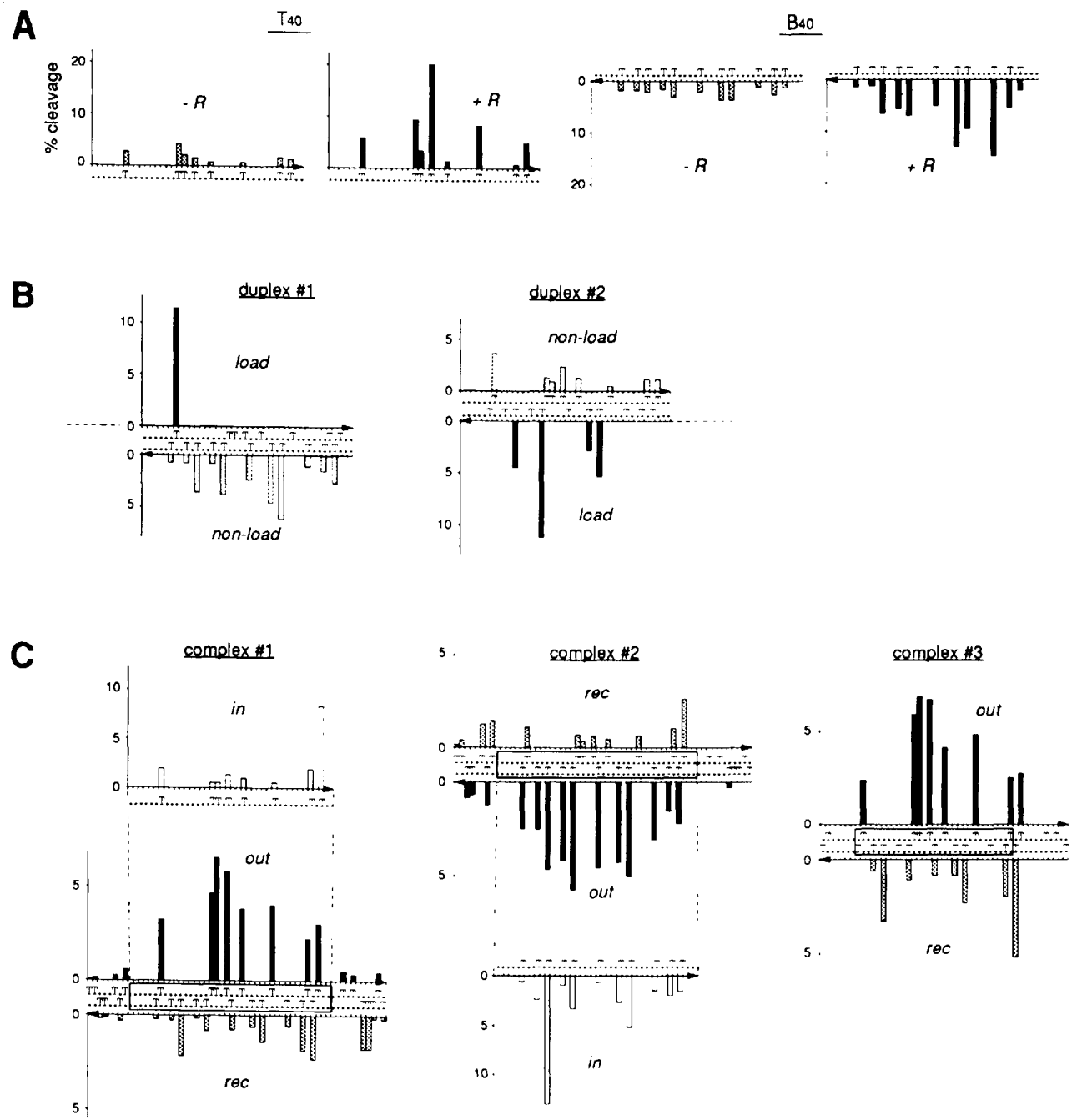

Figure 9. Schematic diagrams of $\mathrm{KMnO}_{4}$ reactivities of various DNA-RecA complexes. The intensity of each band was divided by the sum of the intensities of all bands (percent cleavage; quantified by PhosphorImager, Molecular Dynamics), and the result is plotted against the sequence (bases other than $\mathrm{T}$ are indicated by dots). The patterns of the strands that are identical in sequence to the top strand in Fig. 1A are plotted upward (the $5^{\prime} \rightarrow 3^{\prime}$ polarity is from left to right), and those identical to the bottom strand are plotted downward (polarity is from right to left). (A) ssDNA-RecA complexes $\left(\mathrm{KMnO}_{4}\right.$ concentration, $\left.0.6 \mathrm{mM}\right)$. (Solid and stippled bars) With $(+\mathrm{R})$ and without $(-\mathrm{R}) 3 \mu \mathrm{M}$ RecA, respectively. $(B)$ dsDNA-RecA complexes. (Solid and stippled bars) Loading $\left(\mathrm{KMnO}_{4}\right.$ concentration, $0.4 \mathrm{mM})$ and nonloading $(4.0 \mathrm{mM})$ strands, respectively. Only the patterns observed within the true duplex regions are shown. $(C)$ Synaptic complexes. (Solid bars) Outgoing strands $\left(\mathrm{KMnO}_{4}\right.$ concentration, $\left.2.5 \mathrm{~mm}\right)$; (stippled bars) recipient strands (2.5 mM); open bars, incoming strands $(0.75 \mathrm{mM})$. The homologous regions are boxed. Note that the homologous region in complex 3 is 5 bases shorter at both ends than those in the other two complexes.

chemicals can poison proteins to some extent and, as such, could cause a deformation of the complexes before they modify DNA. These possibilities cannot be ruled out entirely; however, the chemical reactivity patterns presented in this discussion would not have been observed if DMS or $\mathrm{KMnO}_{4}$ completely destroyed the synaptic complex before it attacked the DNA.

How does RecA recognize a homology between ssDNA and dsDNA?

The results described in this discussion do not support the view that formation of a stable triple helix involving
non-Watson-Crick base-pairing accounts for the stable DNA synapsis observed in the presence of ATP $y$ S. These results, however, do not rule out a transient involvement of some unusual modes of base-pairing, especially those that might only exist before the stage analyzed here. Therefore, the following two possibilities remain for the mechanism of homology recognition. The simpler of the two, which the results in this discussion support, is that homology is recognized by Watson-Crick base-pairing; homology recognition and exchange of DNA strands constitute two aspects of a mechanistically identical step. Alternatively, homologous DNAs might be initially discriminated from nonhomologous DNAs by vir- 
tue of their ability to form some kind of transient, but specific contact with the target, possibly including nonWatson-Crick base-pairing. In this regard, however, Jain et al. (1992) recently reported that a dsDNA in which all $\mathrm{G}$ residues are substituted by 7 -deazaguanines (which eliminates the hydrogen-bonding acceptor at the 7 position) is as efficient a target in the strand exchange reaction as unsubstituted dsDNA. These results are not only consistent with the DMS-reactivity data reported here but also argue against the view that the process of homology recognition involves an intermediate in which N-7 of G plays a critical role.

The results in this discussion are not necessarily at odds with those reported by Hsieh et al. (1990) and Rao et al. (1991), who found a stable DNA structure after the removal of RecA in which all three strands appear to be somehow juxtaposed. The apparently different experimental outcome may be simply due to differences in experimental conditions, most notably the combination of DNA substrates used. The experiments in this discussion employed short ssDNA with homology restricted to the middle of the linear target dsDNA, whereas Hsieh and Rao and their coworkers used a long circular ssDNA and a linear dsDNA in which the homology (ranging from 38-56 bp in length in one study to a few kilobases in another) is restricted to the $5^{\prime}$ end of the recipient strand of the dsDNA. When the homology was restricted to the middle or the other end of the dsDNA, the stable, protein-free, three-stranded structure was not detected (Rao et al. 1991), in agreement with the results reported here. If the putative triplex DNA involving non-WatsonCrick base-pairing is one of the stable intermediates of DNA synapsis as proposed (Hsieh et al. 1990; Rao et al. 1991), it will be interesting to investigate why the efficient formation and/or stability of this structure require a particular end of DNA, as this has been unprecedented up to now in canonical DNA triple helices. Alternatively, it seems possible that the strand exchange products in the synaptic complex might, under some condition, collapse with dissociation of RecA, giving rise to some aberrant product. In this regard, it will be also important to examine whether the putative triplex intermediate can really be chased into the normal products.

\section{A homologous DNA end is not essential for local exchange of DNA strands}

Comparison of the chemical reactivity data of the synaptic complex carrying homologous DNA ends with the complex lacking a homologous end indicates that irrespective of the presence or absence of such ends, the base-pairing status is essentially that of the end product of strand exchange. Homology at the end of DNA is thus probably dispensable for the exchange of DNA strands. These results are consistent with the idea that the strand exchange takes place within a RecA complex, in which three DNA strands are held together, by the "swinging" of the bases (rotation orthogonal to the helix axis; Howard-Flanders et al. 1984). (The exact motion of this swinging, however, is not at all clear at this moment; therefore, this should be regarded as an operational term in the following arguments.)

Why, then, is a homologous end needed at all to produce stable strand exchange products? Such an end can act as a swivel that permits axial rotation of the heteroduplex with respect to the axis of the parental duplex. It has been speculated that RecA might actively and directionally promote this axial rotation of DNA at the expense of ATP hydrolysis, which, in turn, would drive the exchange of DNA strands in a unidirectional fashion. This model was invoked in part to explain the observation that global extension of heteroduplex, at least under certain conditions, appears to be unidirectional (Cox and Lehman 1981; Kahn et al. 1981; West et al. 1981). The results described here, however, suggest that the RecAmediated DNA rotation is not the sole mechanism of strand exchange. The requirement for homologous ends can be explained simply by the view that the product $\mathrm{D}$ loop made without a homologous end falls apart rapidly after dissociation of RecA owing to spontaneous branch migration; the strand exchange without a homologous end is simply unproductive rather than nonexistent. Nonetheless, as the length of homology analyzed in this study is rather short ( 30 or $40 \mathrm{bp}$ ), it remains possible that RecA might play a dual role in exchanging DNA strands: one for local strand exchange by the swinging of bases, which requires neither ATP hydrolysis nor homologous DNA ends, and the other for global extension of the heteroduplex, possibly by axial rotation of DNA, which might require both.

\section{How does RecA exchange DNA strands?}

The model for the strand exchange reaction proposed below is primarily based on the finding that a RecA filament carries two nonequivalent DNA-binding sites (Takahashi et al. 1989; Müller et al. 1990). These studies indicate that ssDNA or dsDNA can bind to both sites simultaneously, irrespective of a sequence homology or complementarity la complex holding two dsDNA molecules was not detected, however). Interestingly, characteristics of dsDNA-ssDNA-RecA ternary complexes differ, depending on whether the ssDNA-RecA complex is made first (and then added to a dsDNA) or the dsDNARecA complex is made first (Kubista et al. 1990; Müller et al. 1990). These results support the view that the first DNA, regardless of whether it is ssDNA or dsDNA, binds to a particular site of the two (primary site), which in turn, makes the other site (secondary site) available for another DNA to bind. Consistent with this view, Egelman and $Y u(1989)$ reported that the ssDNA in an ssDNA-RecA complex appears to occupy the same location as that occupied by one of the two strands in a dsDNA-RecA complex. Also in line with this view may be the study by Stasiak et al. (1988), in which the polarity of the RecA filament was determined with respect to the polarity of resident ssDNA. This analysis might pose a paradox if the two DNA-binding sites are truly equivalent, inasmuch as the ssDNA-binding polarity of the two sites should be antiparallel if these sites are also involved 
in the annealing reaction mediated by RecA. In addition to the two DNA-binding sites, Kubista et al. (1990) reported the existence of the third, weak ssDNA-binding site in a RecA filament, a finding that is also taken into account in the model proposed below.

Figure 10 presents the model for a possible mechanism of the DNA strand exchange reaction. This model is similar to the one proposed earlier (Howard-Flanders et al. 1984) in that exchange of DNA strands is envisioned as the swinging of the bases within a RecA filament. Figure 10, however, also contains a new proposal concerning the issue: At which DNA-binding site in the complex might the products be located after local strand exchange? Let us suppose, with some justification as described above, that the incoming ssDNA binds exclusively to the primary site; the presynaptic complex with the "donor" bound to its primary site then searches for a "target" using the open secondary site, whose binding mode must therefore be transient (Fig. 10, steps 1 and 2). After the homology is recognized and strand exchange is accomplished (steps 2 and 3), one can envision essentially three kinds of models for the location of the product heteroduplex. First, the heteroduplex might be bound at the primary site; the recipient strand is transferred from the secondary site to the primary site. The second is the exact opposite; the incoming strand might be transferred from the primary site to the secondary site, so that the heteroduplex becomes associated with the secondary site. The third possibility is that the heteroduplex might be located as a cross bridge between the two sites; two strands comprising a heteroduplex stay at the sites to which they originally bound.

None of the results presented here or our current knowledge of structure-function relationship of RecA can strictly distinguish these possibilities; however, the second model (illustrated in Fig. 10), which assumes the association of the heteroduplex with the secondary site, seems to be more attractive than others. First, upon adding nonhomologous ssDNA, RecA molecules in the synaptic complex appear to be rapidly transferred to the challenging ssDNA [Fig. 3; a similar observation has also been made by Pugh and Cox (1987)]. These observations cannot be explained easily by redistribution of RecA due to rapid equilibrium, as the RecA molecules involved in this complex seem to be essentially immobile by themselves (Fig. 3A). The results, however, can be explained if there is an unoccupied ssDNA-binding site in the complex, to which the challenging ssDNA can bind and promote dissociation of RecA from the resident DNA (Fig. 10 , steps 5 and 6) The cross-bridging model is thus not particularly attractive. Second, paradoxically, it is generally thought that RecA molecules in simple dsDNARecA complexes made in the presence of ATP $\gamma S$ are essentially immobile even in the presence of a challenging ssDNA /also recall that, in Fig. 3B, the bands indicative of dsDNA-RecA complexes, $A+B+C$, were resistant to the ssDNA challenge). Provided that the duplex in dsDNA-RecA complexes is located at the primary site (see abovel, it seems as if DNA binding at the secondary site does not cause rapid dissociation of RecA from the

Figure 10. A model for strand exchange mediated by RecA. Axial views of RecA filaments are shown. The DNA strands are indicated by cir- $^{-}$ cles: (Solid) Incoming strands; (open) recipient strands; (shaded) outgoing strands. The duplex DNA is illustrated as a dumbbell. Brackets surrounding some of the complexes are meant to indicate the potentially transient nature of these complexes. (Step 1) Presynaptic complex formation. The incoming ssDNA binds exclusively to the primary site, which, in turn, makes the secondary site, and possibly the third site, open. (Step 2) Homology search. The potential target duplex binds exclusively to the secondary site. In case of nonhomology, this dsDNA simply dissociates from the secondary site without promoting the dissociation of incoming strand from the primary site; in case of homology, it proceeds to step 3. Although the duplex bound to the secondary site is depicted as if melted, other transient DNA interactions are possible. (Step 3) Local strand exchange resulting in stable DNA synapsis. The heteroduplex is assumed to occupy the secondary site and the displaced ssDNA at the

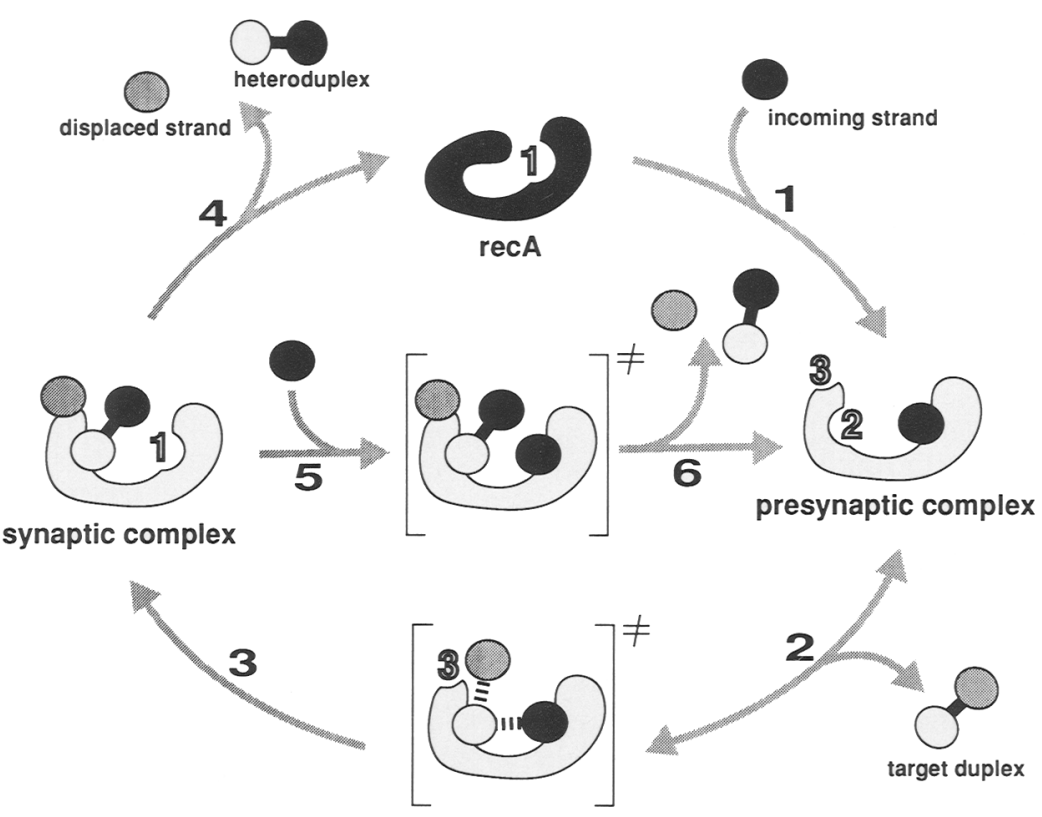
third, weak DNA-binding site. (Step 4) Product release. Several lines of evidences have indicated that release of displaced ssDNA precedes that of the product heteroduplex. This finding discourages certain arrangements of the strand exchange products in a RecA filament (e.g., an arrangement in which the heteroduplex cross-bridges the two sites and the ssDNA is entrapped between this heteroduplex and the inner wall of the RecA filament). (Steps 5,6) A putative pathway of how an additional nonhomologous ssDNA might induce rapid dissociation of RecA from the synaptic complex. 
DNA bound at the primary site, which is also consistent with the potentially transient nature of DNA binding at the secondary site, as postulated above. It is thus simpler to imagine that the putative open site in the synaptic complex is not the secondary site but the primary site. Then, it is a logical requirement to assign the heteroduplex to the secondary site and the locally displaced ssDNA elsewhere, possibly to the third, weak ssDNAbinding site (Kubista et al. 1990), an arrangement that also takes into account that release of displaced ssDNA precedes that of the heteroduplex in the strand exchange reaction (Pugh and Cox 1987). Finally, this arrangement can provide an economical explanation for significant differences observed in $\mathrm{KMnO}_{4}$ reactivities between a heteroduplex in the synaptic complex and a normal duplex in the dsDNA-RecA complex and also between a displaced ssDNA in the synaptic complex and a strand in the ssDNA-RecA complex.

\section{Materials and methods}

\section{DNAs}

All of the ssDNAs used in chemical probing analyses are listed in Figure 1A. The $T_{59}$ used in the characterization of the synaptic complex corresponds to a region at positions 409-467 of pUC18 (GCCTGCA . . CATGGTC, in the polylinker region). These ssDNAs were chemically synthesized and purified by sequencing gel electrophoreses. Duplexes 1 and 2 were made by thermal annealing of the ssDNAs, followed by purification through nondenaturing polyacrylamide gel electrophoresis (PAGE). These duplexes were found to survive ethanol precipitation. Where indicated, the DNA was labeled at the $5^{\prime}$ end by T4-polynucleotide kinase and $\left[\gamma^{32} \mathrm{P}\right] \mathrm{ATP}$ or at the $3^{\prime}$ end by terminal deoxynucleotidyl transferase and $\left[\alpha{ }^{-32} \mathrm{P}\right]$ dideoxy-ATP. $\phi X 174$ viral DNA was purchased from BRL Life Technologies. Concentrations of DNA (nucleotide molar, $\mathrm{M}$ ) are calculated by using extinction coefficients of $1.0 \times 10^{4}$ and $6.7 \times 10^{3} \mathrm{M} / \mathrm{cm}$ at $260 \mathrm{~nm}$ for ssDNA and dsDNA, respectively.

\section{Proteins and chemicals}

RecA was purchased from U.S. Biochemical and further purified by mono-S (Pharmacia LKB) column chromatography. The peak fractions were dialyzed against RecA dilution buffer: $20 \mathrm{mM}$ Tris-acetate $(\mathrm{pH} 7.5$ at $1 \mathrm{M}$ at room temperature), $0.1 \mathrm{mM}$ EDTA, $1 \mathrm{mM}$ dithiothreitol (DTT), 10\% glycerol. The concentration was calculated by using an extinction coefficient of $2.16 \times 10^{4}$ $\mathrm{M} / \mathrm{cm}$ at $278 \mathrm{~nm}$ in the RecA dilution buffer (Kuramitsu et al. 1981). Sources of other proteins are as follows: restriction enzymes (New England Biolabs); T4 polynucleotide kinase and terminal deoxynucleotidyl transferase (Pharmacia LKB); E. coli exonuclease I (Exo I) (U.S. Biochemical); E. coli exonuclease III (Exo III) and phage $\lambda$ exonuclease ( $\lambda$ Exo), (BRL Life Technologies); Calf-intestine phosphatase (CIP) (Boehringer Mannheim). Sources of ehemicals are as follows: DMS, hydrazine, and piperidine (Aldrich); $\mathrm{KMnO}_{4}$ (Fluka); ATP $\gamma \mathrm{S}$ (Boehringer Mannheim); $\left[\gamma^{-32} \mathrm{P}\right] \mathrm{ATP}$ (New England Nuclear); $\left[\alpha^{-32} \mathrm{P}\right]$ dideoxyATP (Amersham).

\section{Formation of synaptic complex}

The standard reaction mixture to make the synaptic complex contained $9.3 \mathrm{nM}$ (in molecule; $50 \mu \mathrm{M}$ in nucleotide) of ScaI- linearized pUC18 DNA and $18.6 \mathrm{nM}$ ssDNA $(0.74-1.9 \mu \mathrm{M}$ in nucleotide, depending on the length) in the buffer consisted of $40 \mathrm{~mm}$ Tris-acetate ( $\mathrm{pH} 7.5), 100 \mathrm{~mm}$ potassium acetate, $9 \mathrm{~mm}$ magnesium acetate, $1 \mathrm{mM}$ DTT, $250 \mu \mathrm{M}$ ATP $\gamma \mathrm{S}$, and $100 \mu \mathrm{g}$ of bovine serum albumin (BSA) per $\mathrm{ml}$. The concentrations of RecA were optimized for each incoming ssDNA as described in the text, and were $0.68 \mu \mathrm{M}$ and $1.0 \mu \mathrm{M}$ for 40 -mer (or $59-\mathrm{mer}$ ) and 100-mer ssDNAs, respectively. The reaction was initiated by the addition of one-tenth volume of RecA (appropriately diluted in RecA dilution buffer), followed by incubation at $37^{\circ} \mathrm{C}$ for $1 \mathrm{hr}$. Formation of the synaptic complex was assayed by adding $1.5 \mu \mathrm{l}$ of RsaI [10 U/Ml in RsaI dilution buffer: $10 \mathrm{mM}$ Tris- $\mathrm{HCl} / \mathrm{pH}$ 7.4), $0.1 \mathrm{~mm}$ EDTA, $50 \mathrm{~mm} \mathrm{KCl}, 1 \mathrm{mM}$ DTT, $50 \%$ glycerol, 200 $\mu \mathrm{g}$ of BSA per ml] to the $25 \mu \mathrm{l}$ of the reaction mixture, followed by an additional incubation at $37^{\circ} \mathrm{C}$ for, normally, $5 \mathrm{~min}$. In the ssDNA challenge experiments, the synaptic complex was made at $37^{\circ} \mathrm{C}$ for $1 \mathrm{hr}$ as described above and then $3 \mathrm{~mm}$ (in nucleotide) $\phi \mathrm{X}$ ssDNA was added to a final concentration of $120 \mu \mathrm{M}$. The incubation was continued at $37^{\circ} \mathrm{C}$ for various time periods, followed by digestion with RsaI as described above.

\section{Isolation of $D$ loops}

Isolation of $\mathrm{D}$ loops was carried out by use of a spin column, which was made in a $1-\mathrm{ml}$ syringe containing $0.8 \mathrm{ml}$ of Sephacryl S-300 superfine (Pharmacia LKB) in $10 \mathrm{~mm}$ Tris- $\mathrm{HCl}$ (pH 7.5 at $\mathrm{I} \mathrm{M}$ at room temperature) and $1 \mathrm{~mm}$ EDTA. The column was prespun at $300 \mathrm{~g}$ for $4 \mathrm{~min}$ before loading the samples. The mixture $(150 \mu l)$ containing $D$ loops was loaded onto the prespun column and centrifuged at $300 \mathrm{~g}$ at room temperature for $5 \mathrm{~min}$. The spin column treatment was repeated twice. Under these conditions, recoveries of large DNAs (including D loops) and short ssDNAs at each round were $\sim 70 \%$ and $5 \%$, respectively, as judged from gel electrophoreses of the samples. The final sample thus contains negligible amounts of free ssDNA, with $\sim 50 \%$ recovery of $D$ loops.

\section{Digestion of $D$ loops by EcoRI}

The synaptic complex was formed between supercoiled pUC18 DNA and 5' end-labeled $T_{59}$. The deproteinized joint molecule (D loop) was purified by the spin column method, as described above, and digested by EcoRI. Nonlabeled pBR322 supercoiled DNA was also included in the digestion mixture as an internal control. Aliquots were taken at various time points and run on agarose as well as sequencing gels. The cleavage efficiency was calculated by quantifying the disappearance of the radioactive band that comigrated with supercoiled pUC18 in the agarose gel and also by quantifying the radioactivity of cleaved $T_{59}$ in the sequencing gel. The sum of radioactivities of the cleaved and uncleaved $T_{59}$ was constant at each time point. The cleavage time course of the control pBR322 DNA was quantified by densitometric analysis of the photographic negative of ethidiumstained gels.

\section{Chemical probing of synaptic complex: target duplex}

To examine chemical reactivities of the target duplex within the synaptic complex (recipient and outgoing strands), the complex was made by incubating ScaI-linearized pUC18 DNA and short ssDNA with RecA in $200 \mu \mathrm{l}$ of standard reaction mixture described above. After a 1 -hr incubation at $37^{\circ} \mathrm{C}, 12 \mu \mathrm{l}$ of $\mathrm{RsaI}$ $(10 \mathrm{U} / \mu \mathrm{l})$ was added to the reaction mixture and incubation was continued for another $5 \mathrm{~min}$. To each sample, $5 \mu \mathrm{l}$ of $\mathrm{KMnO}_{4}$ (freshly dissolved in water to appropriate concentrations) or DMS (appropriately diluted with ethanol before use) was added, 
followed by additional incubation at $37^{\circ} \mathrm{C}$ for $2 \mathrm{~min}$. The reaction was quenched by adding $50 \mu$ of standard DMS stop solution (Maxam and Gilbert 1980), to which $750 \mu$ l of chilled ethanol was immediately added. The samples were collected by centrifugation, suspended in $250 \mu \mathrm{l}$ of $0.3 \mathrm{M}$ sodium acetate $(\mathrm{pH}$ 7.0) at $0^{\circ} \mathrm{C}$, extracted with phenol/chloroform $(1: 1)$, and ethanol precipitated. Resuspended samples were then digested with HinPI to completion and fractionated by nondenaturing polyacrylamide gel electrophoresis. A 130-bp HinPI fragment, which includes the protected $R s a I$ site, was purified from the gel by the "crush and soak" method. The $\mathrm{KMnO}_{4}$-modified fragment was processed by the standard piperidine cleavage protocol $/ 1 \mathrm{M}$ at $90^{\circ} \mathrm{C}$ for $30 \mathrm{~min} ;$ Maxam and Gilbert 1980). To enhance the cleavage of methylated $\mathrm{C}$, the DMS-modified fragment was treated with hydrazine according to the method described by Cowing et al. \{1989) before the piperidine cleavage. A negative control (without RecA and ssDNA) was processed exactly the same way, except that the RsaI dilution buffer was added instead of the enzyme solution. Taking each of the RsaI protection efficiencies into consideration $(\sim 50 \%)$, amounts that were calculated to be roughly equivalent to 0.2 pmole of the HinPI 130-bp fragment were loaded onto an $8 \%$ sequencing gel. After electrophoresis, DNA in the gel was electrotransferred to GeneScreen (Dupon-NEN), followed by UV cross-linking. The chemical reactivity pattern was obtained by hybridization with ${ }^{32}$ P-labeled strand-specific probe, as described by Church and Gilbert (1984), with minor modifications (Richet et al. 1986).

\section{Chemical probing of synaptic complex: incoming strand}

To examine chemical reactivities of the incoming strand of the synaptic complex, supercoiled pUC18 DNA and 5' end-labeled ssDNA, $T_{40}$ or $B_{40}$, were used to make the complex in $100 \mu l$ of a standard reaction mixture as described above. After formation of the complex, samples were treated with DMS or $\mathrm{KMnO}_{4}$ for $2 \mathrm{~min}$ at $37^{\circ} \mathrm{C}$ and quenched by adding $25 \mu \mathrm{l}$ of DMS stop solution. To these samples, EDTA and SDS were immediately added to final concentrations of $20 \mathrm{~mm}$ and $0.5 \%$, respectively, from which the resulting D loop was isolated by the spin col. umn method. Treatment of the synaptic complex with DMS or $\mathrm{KMnO}_{4}$, for some unknown reasons, tended to increase the recovery of the D loops (about twofold). The purified D loop was cleaved by the same procedure as described above, and the samples were run on an $18 \%$ sequencing gel, followed by autoradiography. The naked ssDNA control in Figure 7 was carried out in essentially the same way except for the absence of RecA (without spin column treatment). Another control, chemical probing of the ssDNA within protein-free D loops, was done by first isolating the $\mathrm{D}$ loops and then treating the samples with a chemical; further purification of the chemically treated D loops was not done.

\section{Chemical probing of SsDNA-RecA complexes}

The ssDNA-RecA complex was made under the same conditions as used for synaptic complex formation, except for the absence of dsDNA, with the $5^{\prime}$ end-labeled $\mathrm{T}_{40}$ or $\mathrm{B}_{40}(0.74 \mu \mathrm{M})$ in $25 \mu \mathrm{l}$ of reaction mixture /the RecA concentrations were indicated in the legend to Fig. 2). To assess the complex formation, some samples were treated with $1 \mu \mathrm{l}$ of Exo $[(5 \mathrm{U} / \mu \mathrm{l})$, which digests an ssDNA in a $5^{\prime} \rightarrow 3^{\prime}$ polarity, or a phosphatase $\mathrm{CIP}\{1 \mathrm{U} / \mu \mathrm{l}\}$ at $37^{\circ} \mathrm{C}$ for $10 \mathrm{~min}$ before the chemical modification by DMS or $\mathrm{KMnO}_{4}$. Without RecA, virtually no radioactivity can be recovered by ethanol precipitation after these enzyme treatments. In the presence of $1-3 \mu \mathrm{M}$ RecA, most of the ssDNAs were resistant to Exo I but only partially $(5-20 \%)$ re- sistant to CIP, suggesting that the extreme $5^{\prime}$ ends were not stably bound by RecA under these conditions. These concentrations of RecA are much higher than those expected from known stoichiometry of the DNA binding of RecA lone RecA per 3 bases). Leahy and Radding (1986) also observed that in the case for short ssDNAs, the binding of RecA is not stoichiometric even in the presence of ATP $\gamma$ S.

\section{Chemical probing of dsDNA-RecA complexes}

To make the dsDNA-RecA complex, $\sim 20 \mathrm{~nm}$ (in molecule; $-2.2 \mu \mathrm{M}$ in nucleotide) of duplex 1 or 2 , which was labeled at either the 3 ' ends of loading strands or the $5^{\prime}$ ends of nonloading strands was incubated with $1.8 \mu \mathrm{M}$ RecA protein at $37^{\circ} \mathrm{C}$ for 30 min under the same conditions as used for synaptic complex formation. To this mixture $(50 \mu l), 2.5 \mu \mathrm{l}$ of $R s a \mathrm{I}(10 \mathrm{U} / \mu \mathrm{l})$ was added, and incubation was continued for another $30 \mathrm{~min}$; the mixture was then treated with DMS or $\mathrm{KMnO}_{4}$ for 2 min (probably because of the shortness of the duplex, the condition of Rsal digestion had to be very harsh to achieve a complete digestion without RecA). No significant denaturation of the duplex was detected during the total 1-hr incubation with RecA and Rsal, according to the assay using polyacrylamide gel electrophoresis. The Rsal-resistant duplex fragments, which constituted $>90 \%$ of input duplexes under these conditions, were purified by nondenaturing polyacrylamide gel electrophoresis, chemically cleaved, and run on a sequencing gel. The efficient formation of the complex was also verified separately by confirming the resistance to Exo III, which digests a dsDNA in a $3^{\prime} \rightarrow 5^{\prime}$ polarity, and/or to $\lambda$ Exo, which digests a dsDNA in a $5^{\prime} \rightarrow 3^{\prime}$ polarity.

\section{Acknowledgments}

I am grateful to Drs. Regis Krah, Fraser McBlane, Tania Baker, Gary Felsenfeld, Joanne Hesse, Martin Gellert, Kiyoshi Mizuuchi, Howard Nash, and Joseph Menetski of the National Institutes of Health (NIH) for their critical reading of the manuscript, and to Dr. Frederic Bushman (NIH) for his critical reading and editing of the manuscript. I thank Dr. Michael Cox et al. (University of Wisconsin) for providing me with a preprint of their manuscript, and Drs. Moon Kim and Daniel CameriniOtero (NIH) for stimulating discussions. I express my special thanks to Dr. Kiyoshi Mizuuchi for his support and encouragement, without which this study would not have been possible.

The publication costs of this article were defrayed in part by payment of page charges. This article must therefore be hereby marked "advertisement" in accordance with 18 USC section 1734 solely to indicate this fact.

\section{References}

Bianchi, M., C. DasGupta, and C.M. Radding. 1983. Synapsis and the formation of paranemic joints by E. coli RecA protein. Cell 34: 931-939.

Bortner, C. and I. Griffith. 1990. Three-stranded paranemic joints: Architecture, topological constraints and movement. I. Mol. Biol. 215: 623-634.

Cassuto, E. and P. Howard-Flanders. 1986. The binding of RecA protein to duplex DNA molecules is directional and is promoted by a single stranded region. Nucleic Acids Res. 14: $1149-1157$.

Chow, S.A., S.M. Honigberg, and C.M. Radding. 1988. DNase protection by recA protein during strand exchange: Asymmetric protection of the holliday structure. /. Biol. Chem. 
263: 3335-3347.

Church, G.M. and W. Gilbert. 1984. Genomic sequencing. Proc. Natl. Acad. Sci. 81: 1991-1995.

Collier, D.A., J.-L. Mergny, N.T. Thuong, and C. Helene. 1991. Site-specific intercalation at the triplex-duplex junction induces a conformational change which is detectable by hypersensitivity to diethylpyrocarbonate. Nucleic Acids Res. 1: 4219-4224.

Cowing, D.W., J. Mecsas, M.T. Record Jr. and C.A. Gross. 1989. Intermediates in the formation of the open complex by RNA polymerase holoenzyme containing the sigma factor $\sigma^{32}$ at the groE promotor. I. Mol. Biol. 210: 521-530.

Cox, M.M. and I.R. Lehman. 1981. Directionality and polarity in recA protein promoted branch migration. Proc. Natl. Acad. Sci. 78: 6018-6022.

- 1987. Enzymes of genetic recombination. Annu. Rev. Biochem. 56: 229-262.

DasGupta, C., T. Shibata, R.P. Cunningham, and C.M. Radding. 1980. The topology of homologous pairing promoted by RecA protein. Cell 22: 437-446.

DiCapua, E. and B. Müller. 1987. The accessibility of DNA to dimethylsulfate in complexes with RecA protein. EMBO /. 6: $2493-2498$

Egelman, E.H. and A. Stasiak. 1986. The structure of helical RecA-DNA complexes. I. Complexes formed in the presence of ATP $y$ S or ATP. J. Mol. Biol. 191: 677-697.

Egelman, E.H. and X. Yu. 1989. The location of DNA in RecADNA helical filaments. Science 245: 404-407.

Flory, J., S.S. Tsang, and K. Muniyappa. 1984. Isolation and visualization of active presynaptic filaments of RecA protein and single-stranded DNA. Proc. Natl. Acad. Sci. 81: 70267030 .

Hanvey, J.C., M. Shimizu, and R.D. Wells. 1990. Site-specific inhibition of EcoRI restriction/modification enzymes by a DNA triple helix. Nucleic Acids Res. 18: 157-161.

Hayatsu, H. and T. Ukita. 1967. The selective degradation of pyrimidines in nucleic acids by permanganate oxidation. Biochem. Biophys. Res. Commun. 29: 556-561.

Honigberg, S.M., D.K. Gonda, J. Flory, and C.M. Radding. 1985. The pairing activity of stable nucleoprotein filaments made from RecA protein, single-stranded DNA, and adenosine 5'( $\gamma$-thio)triphosphate. J. Biol. Chem. 260: 11845-11851.

Howard-Flanders, P., S.C. West, and A. Stasiak. 1984. Role of RecA protein spiral filaments in genetic recombination. $\mathrm{Na}$ ture 309: 215-220.

Hsieh, P., C.S. Camerini-Otero, and R.D. Camerini-Otero. 1990. Pairing of homologous DNA sequences by proteins: Evidence for three-stranded DNA. Genes \& Dev. 4: 1951-1963.

lida, S. and H. Hayatsu. 1971. The permanganate oxidation of deoxyribonucleic acid. Biochem. Biophys. Acta 240: 370375.

Jain, S.K., R.B. Inman, and M.M. Cox. 1992. The putative threestranded DNA pairing intermediate in RecA protein-mediated DNA strand-exchange: No role for guanine N-7. $/$. Biol. Chem. 267: 4215-4222.

Kahn, R., R.P. Cunningham, C. DasGupta, and C.M. Radding. 1981. Polarity of heteroduplex formation promoted by Escherichia coli RecA protein. Proc. Natl. Acad. Sci. 78: 47864790.

Kowalczykowski, S.C. 1991. Biochemistry of genetic recombination: Energetics and mechanism of DNA strand exchange. Annu. Rev. Biochem. Biophys. Chem. 20: 539-575.

Kubista, M., M. Takahashi, and B. Norden. 1990. Stoichiometry, base orientation, and nuclease accessibility of RecADNA complexes seen by polarized light in flow-oriented solution: Implications for the mechanism of genetic recombi- nation. I. Biol. Chem. 265: 18891-18897.

Kuramitsu, S., K. Hamaguchi, T. Ogawa, and H. Ogawa. 1981. A large-scale preparation and some physicochemical properties of recA protein. J. Biochem. 90: 1033-1045.

Leahy, M.C. and C.M. Radding. 1986. Topography of the interaction of RecA protein with single-stranded deoxyoligonucleotides. I. Biol. Chem. 261: 6954-6960.

Lindsley, I.E. and M.M. Cox. 1989. Dissociation pathway for recA nucleoprotein filaments formed on linear duplex DNA. 1. Mol. Biol. 205: 695-711.

Maher, L.J., III, B. Word, and P.B. Dervan. 1989. Inhibition of DNA binding proteins by oligonucleotide-directed triple helix formation. Science 245: 725-730.

Maxam, A. and W. Gilbert. 1980. Sequencing end-labeled DNA with base-specific chemical cleavages. Methods Enzymol. 65: 499-560.

Menetski, J.P. and S.C. Kowalczykowski. 1985. Interaction of recA protein with single-stranded DNA: Quantitative aspects of binding affinity modulation by nucleotide cofactors. I. Mol. Biol. 181: 281-295.

-1987. Transfer of recA protein from one polynucleotide to another: Effect of ATP and determination of the processibity of ATP hydrolysis during transfer. I. Biol. Chem. 262: 2093-2100

Menetski, J.P., D.G. Bear, and S.C. Kowalczykowski. 1990. Stable DNA heteroduplex formation catalyzed by the Escherichia coli RecA protein in the absence of ATP hydrolysis. Proc. Natl. Acad. Sci. 87: 21-25.

Müller, B., T. Koller, and A. Stasiak. 1990. Characterization of the DNA binding activity of stable RecA-DNA complexes: Interaction between the two DNA binding sites within RecA helical filaments. J. Mol. Biol. 212: 97-112.

Pugh, B.F. and M.M. Cox. 1987. RecA protein binding to the heteroduplex product of DNA strand exchange. $I$. Biol. Chem. 262: 1337-1343.

Radding, C.M. 1988. Homologous pairing and strand exchange promoted by Escherichia coli RecA protein. In Genetic recombination, (ed. R. Kucherlapati and G.R. Smith), pp. 193229. American Society for Microbiology, Washington, D.C.

Rao, B.J., M. Dutreix, and C.M. Radding. 1991. Stable threestranded DNA made by RecA protein. Proc. Natl. Acad. Sci. 88: 2984-2988.

Register J.C., III, G. Christiansen, and J. Griffith. 1987. Electron microscopic visualization of the RecA protein-mediated pairing and branch migration phases of DNA strand exchange. I. Biol. Chem. 262: 12812-12820.

Richet, E., P. Abcarian, and H.A. Nash. 1986. The interaction of recombination proteins with supercoiled DNA: Defining the roles of supercoiling in lambda integrative recombination. Cell 46: 1011-1021

Rosselli, W. and A. Stasiak. 1990. Energetics of RecA-mediated recombination reactions: Without ATP hydrolysis RecA can mediate polar strand-exchange but is unable to recycle. $/$. Mol. Biol. 216: 335-352.

Shaner, S.L. and C.M. Radding. 1987. Translocation of Escherichia coli RecA protein from a single-stranded tail to contiguous duplex DNA. I. Biol. Chem. 262: 9211-9219.

Stasiak, A. and E.H. Egelman. 1987. RecA protein-DNA interactions in recombination. In DNA replication and recombination (ed. T. Kelly and T. McMacken), pp. 619-628. Alan R Liss, New York.

. 1988. Visualization of recombination reactions. In Genetic recombination (ed. R. Kucherlapati and G.R. Smith), pp. 265-307. American Society for Microbiology, Washington, D.C.

Stasiak, A., E.H. Egelman, and P. Howard-Flanders. 1988. Struc- 
ture of helical RecA-DNA complexes, III: The structural polarity of RecA filaments and functional polarity in the RecAmediated strand-exchange reaction. I. Mol. Biol. 202: 659662.

Takahashi, M., M. Kubista, and B. Norden. 1989. Binding stoichiometry and structure of RecA-DNA complexes studies by flow linear dichroism and fluorescence spectroscopy: Evidence for multiple heterogeneous DNA co-ordination. /. Mol. Biol. 205: 137-147.

Tsang, S.S., K. Muniyappa, E. Azhderian, D.K. Gonda, C.M. Radding, J. Flory, and J.W. Chase. 1985. Intermediates in homologous pairing promoted by RecA protein: Isolation and characterization of active presynaptic complexes. J. Mol. Biol. 185: 295-309.

Umlauf, S.W., M.M. Cox, and R.B. Inman. 1990. Triple helical DNA pairing intermediates formed by RecA protein. J. Biol. Chem. 265: 16898-16912.

West, S.C., E. Cassuto, and P. Howard-Flanders. 1981. Heteroduplex formation by RecA protein: Polarity of strand-exchanges. Proc. Natl. Acad. Sci. 78: 6149-6153. 


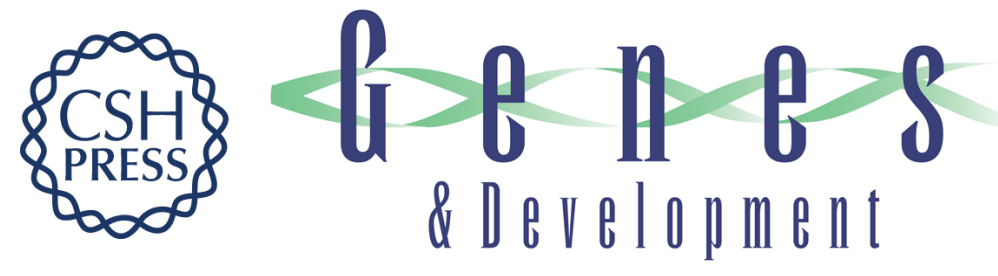

\section{Stable synapsis of homologous DNA molecules mediated by the Escherichia coli RecA protein involves local exchange of DNA strands.}

K Adzuma

Genes Dev. 1992, 6:

Access the most recent version at doi:10.1101/gad.6.9.1679

References This article cites 44 articles, 20 of which can be accessed free at: http://genesdev.cshlp.org/content/6/9/1679.full.html\#ref-list-1

License

Email Alerting Service

Receive free email alerts when new articles cite this article - sign up in the box at the top right corner of the article or click here.

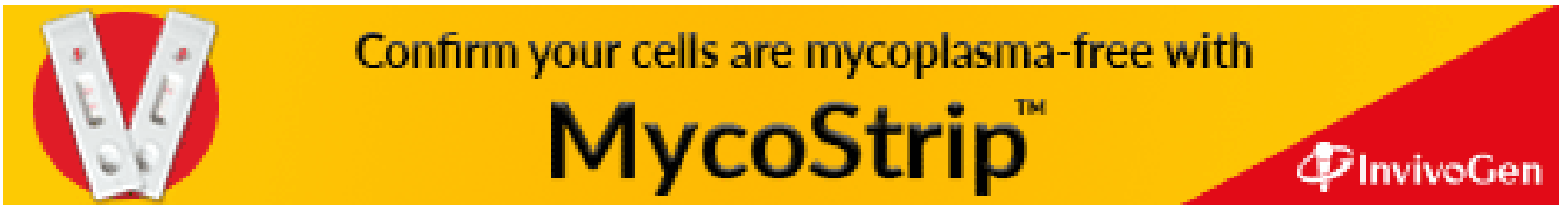

